\title{
Responses of Soil Microbial Communities to the Invasion of Fusarium Oxysporum and Screening for Fusarium Antagonistic Microorganisms From Pathogenic Soil
}

\section{Yehan Tian}

Shandong Agricultural University College of Plant Protection

Kexiang Gao ( $\nabla$ kga063@163.com )

Shandong Agricultural University https://orcid.org/0000-0001-5592-3986

\section{Research}

Keywords: Fusarium oxysporum, microbial community structure, co-culture, Talaromyces purpurogenus, biocontrol mechanisms

Posted Date: February 24th, 2021

DOl: https://doi.org/10.21203/rs.3.rs-227497/v1

License: (c) (i) This work is licensed under a Creative Commons Attribution 4.0 International License. Read Full License 


\section{Abstract}

Background

Fusarium oxysporum is known as a biological pollutant in the farmland soil, that causes vascular wilt on a wide range of cash crops, which is the most destructive disease of plant worldwide. F. oxysporum is a fungus that can colonize and grow saprophytically in plant debris and increase pathogen viability in soil. It is almost impossible to remove once successful colonization. How to effectively reduce the pathogen population and restore the beneficial microbial community in soil is an important subject to control the Fusarium wilt. The main objective of this study is to explore influences of $F$. oxysporum invasion on the microbial community and to screen efficient biocontrol agents from microorganisms with changing relative abundance.

Results

In this study, we evaluated that responses of the soil microbial communities to invasion of $F$. oxysporum. We found that $F$. oxysporum invasion could modify soil physical-chemical properties, soil enzymes activities, microbial community structure and diversity of the soil microbes. We screened and identified an efficient biocontrol strain, Talaromyces purpurogenus Q2, which could be used for defense and suppression of F. oxysporum in bitter gourd and efficiently decreasing incidence of the Fusarium wilt of bitter gourd in the greenhouse and in the field. The research results of biocontrol mechanisms showed that $T$. purpureogenus strain Q2 involved in the resistance of soil microbial communities to invasion of $F$. oxysporum, and the density of $F$. oxysporum was inhibited by exogenous T. purpureogenus and the resident microbes that strain Q2 promoted their growth. In the co-culture of strain Q2 and $F$. oxysporum, we further observed that $T$. purpurogenus Q2 could significantly suppress the mycelia growth and development of $F$. oxysporum by secreting cell wall degrading enzymes, and hinder cell wall formation by suppressing the gene expression of cell wall biosynthesis, such as FomCFEM that is essential for full virulence and stress tolerance in F. oxysporum.

Conclusion

Together we screened a efficient biocontrol agents from microorganisms with resistance to the invasion of $F$. oxysporum. The mechanisms of Talaromyces purpurogenus strain Q2 protected bitter gourd from F. oxysporum is to reduce the relative abundance of rhizospheric $F$. oxysporum by inhibiting the growth and development of F. oxysporum. This work can provide a new biocontrol agent for biocontrol of Fusarium wilt and soil-born diseases of other horticultural crops.

\section{Background}

The effect of Fusarium wilt on the yield and quality of the crops is a hot-investigated topic, as is the effect of their pathogens on the soil microbial communities in which they are occurrence. The microecological impact and control strategy of Fusarium oxysporum is still an unresolved matter, and the interaction between F. oxysporum and soil organisms is an important point to investigate and define.

F. oxysporum is known as a biological pollutant in farmland soil, that causes vascular wilt on a wide range of cash crops, which is the most destructive disease of plant worldwide, and lead to its global loss increasing annually [1, 2]. It comprises more than 100 host-specific strains (formae speciales), many of which have worldwide distribution [3, 4]. The continuous cropping of a single plant in the same field is an important factor that contributes to the successful colonization and accumulation of $F$. oxysporum in soil and leads to the build-up of pathogen populations, reduction of the number and/or activity of beneficial bacteria, with a loss of soil suppressiveness to Fusarium wilt, a decline in soil quality and a severe yield decline [5, 6]. F. oxysporum mainly lives in the soil in the form of dormant chlamydospores, which can survive in the soil for 10-15 years without a host [4, 7, 8, 9]. It can be transmitted by media such as soil, water, agricultural tools and human beings and will become the primary infection source of the disease in the coming year $[9,10]$. The incursion of $F$. oxysporum is hard to detect in the incubation period of the disease. Host root exudates trigger the germination of chlamydospores and there are constantly produced once the host is invaded [11, 12]. Meanwhile, F. oxysporum is a saprophytic fungus that can colonize and grow saprophytically in plant debris and increases pathogen viability in the soil. It is almost impossible to remove once successful colonization [10]. Consequently, how to effectively reduce the pathogen population and restore the healthy soil microbial community is an important subject to control the Fusarium wilt.

Currently, the global need for quality food will continue to increase, how to protect crop plants from diverse diseases and pests without heavily relying on synthetic pesticides will face multiple complex challenges [13]. Although, biocontrol does not achieve the same level of protection of the crops as synthetic pesticides do and faces the problem of having inconsistent results when used in field scale, the use of biocontrol microorganisms for an environmentalfriendly control method of diseases become the most promising and important strategies [14]. Simultaneously, the various biocontrol agents are used to control Fusarium wilt, such as Trichoderma spp., Streptomyces spp. and Bacillus spp. $[15,16,17,18]$. Several studies of biocontrol agents have been reported combination treatment methods using two or more of these agents are more effective in managing the disease than treatment using a single biocontrol agent. At present, the most effective management for controlling Fusarium wilt disease is soil fumigation using chemical fungicides cooperate with bio-organic fertilizer, the Fusarium wilt has been suppressed by reducing the number of chlamydospores of pathogens and re-building the soil microbial community [5].

Therefore, the main objective of this study was to explore the influences of the invasion of $F$. oxysporum on the microbial community and to screen biocontrol agents from microorganisms with changing relative abundance. In order to obtain more accurate test evidence, we built a model of Fusarium wilt of bitter gourd by artificially adding the F. oxysporum f. sp. Momordica (ACCC 39204) and continuous cropping of bitter gourd for 5 years. Based on this model, we evaluated the responses of soil microbial communities to the invasion of $F$. oxysporum and screened out a new Talaromyces sp. with biological control potential, and investigated the control efficacy and control mechanism of the Talaromyces sp. on the Fusarium wilt. Furthermore, the effects of Talaromyces sp. on the metabolic pathways and related gene expression of F. oxysporum during interactions between Talaromyces sp. and F. oxysporum were studied and described, and the effects of these genes on the growth, development, and pathogenicity of $F$. oxysporum were clarified.

Page $2 / 20$ 


\section{Materials And Methods}

\subsection{Fugal strains and plant material}

The fungal pathogens Fusarium oxysporum f. sp. momordicae (Fom) strain SG-15 (Agricultural Culture Collection of China with accession ACCC 39204 ) was isolated from diseased plant of bitter gourd wilt in Shouguang, Shandong Province in P. R. China. Other fungal pathogens besides Fom, such as Fusarium graminearum, F. moniliforme, F. oxysporum f. sp. cucumarinum, F. oxysporum f. sp. niveum, Pyriculariaoryzae, Trichothecium roseum, Cryphonectria parasitica, Cytospora chrysosperma, Phytophthora parasitica, Rhizoctonia solani, used in this study were preserved in our laboratory. The bitter gourd cultivar is Ruyu-41.

\subsection{Study sites and soil sample collection}

We collected soil samples (the standing plant was bitter gourd) at a long-term experimental station of the Shandong Agricultural University, in Taian of Shandong Province, China, August to November 2019. The artificial disease nursery of Fusarium wilt was established in the experimental station in 2015 to investigate the disastrous mechanisms and control strategies of exogenetic invasive F. oxysporum. The incidence of Fusarium wilt of bitter gourd is $100 \%$ from 2017 to 2019. Three treatments with five replicates were set up, including healthy soil without Fusarium inoculation (Control), pathogenic soil with Fusarium inoculation (S-FOM), and the pathogenic soil with Fusarium inoculation was treated with antagonistic fungus (TP). We collected 1 kg soil samples from the rhizosphere of bitter gourd in each plot. The collected soil samples were sieved $(2 \mathrm{~mm})$. A part of the soil for isolation and screening of beneficial microorganisms, a part of the soil for DNA extraction was stored at $-80{ }^{\circ} \mathrm{C}$, and the rest soil for soil fertility was stored at $4{ }^{\circ} \mathrm{C}$. The soil physicochemical characterizations were measured based on the norm system, the soil enzyme activities (i.e. soil fluorescein diacetate hydrolase, soil urease, soil polyphenol oxidase and soil acid phosphatase) were measured according to instructions of the kit (Beijing Solarbio Science \& Technology Co., Ltd.).

\subsection{Isolation of biocontrol agent from the pathogenic soil}

Approximately $10 \mathrm{~g}$ soil was incubated in $100 \mathrm{~mL}$ of sterile distilled water and constantly shaked at $200 \mathrm{rpm}$ for $30 \mathrm{~min}$. $0.2 \mathrm{~mL}$ diluted soil extract was plated on PDA medium Petri plates. Plates were then incubated at $28^{\circ} \mathrm{C}$ for $3-5$ days and the growing colonies were transferred to new Petri dishes with PDA medium in order to establish bacterial or fungal pure cultures. More than 100 colonies were collected and used in subsequent screenings. All microbial collections isolated from the pathogenic soil were tested for antagonism against Fom. Antagonistic examination of microbial isolates against Fom was performed in vitro using PDA plates. Agar disks of Fom was applied on the Petri dish $6 \mathrm{~cm}$ apart from each microbial culture spot. The Petri dishes were then incubated at $28^{\circ} \mathrm{C}$ for 7 days. The percentage of mycelia growth inhibition I (\%) by isolated strain was calculated according to the following formula: I (\%) $=(1$ - Dn/Do) $\times 100$, where I (\%) represents the mean inhibition of mycelia growth, Dn represent the mean diameter of fungal pathogens growth in the presence of isolated strain, and Do represents the mean diameter of fungal pathogens growth in the absence of isolated strain (Control). 14 microbial isolates showing high Fom inhibition were named SK1-SK6, KG-1, Q1-Q2, and M1-M5 (Supplementary Table S1).

\subsection{Morphological and molecular identification of Talaromyces sp. strain Q2}

Talaromyces sp. strain Q2 was inoculated and grown on Petri dishes $(90 \mathrm{~mm})$ with potato dextrose agar (PDA) medium at $25^{\circ} \mathrm{C}$ in the dark, which were performed in triplicate. 2-5 days later, the morphology observation of conidiophores and conidia was performed under Nikon Eclipse $90 \mathrm{i}$ microscope (Nikon, Tokyo, Japan). The pure strains were incubated on different media including Czapek agar (CA), Czapek yeast extract agar (CYA), Sabouraud maltose agar (SMA), Corn meat agar (CMA), Martin media (MM), Sabouraud sucrose agar (SSA), Sabouraud dextrose agar (SDA), Oatmeal agar (OA) at $25^{\circ} \mathrm{C}$ for 7 days, and the colony morphology and microscopic characters were examined, measured and photographed. Morphological identification of Talaromyces sp. strain Q2 was conducted according to the references of Visagie and Su [19, 20]. $\beta-1,3$-glucanase and chitinase produced by strains Q2 were determined qualitatively by the clear zone method on $\beta$-D-glucan and colloidal chitins-containing agar plate. At the same time, we also tested the changes of the enzyme-producing ability of strain Q2 induced by the different substrate. The $\beta$-1, 3-glucanase and chitinase were assessed quantitatively by chitinase enzyme Activity Determination Kit and $\beta$-1, 3-glucanase enzyme Activity Determination Kit (Beijing Solarbio Biotechnology Co., Ltd.), respectively.

Fungal mycelium was harvested from the surface of PDA medium and frozen by liquid nitrogen. Then the DNA samples of strain Q2 were extracted using the Fungal Genomic DNA Kit (BioTeKe Biotech Co. Ltd, Beijing, China) according to the manufacturer's instructions and stores at -20 ${ }^{\circ} \mathrm{C}$. Primers of ITS1/ITS4, $\mathrm{Bt2a} / \mathrm{Bt2b}$, and cmdAD1/cmdQ1 were used to identify the strain Q2 [21, 22, 23]. The primers, PCR program, and the reference sequence used in the current study have been described in the Supplementary Table S2. The genomes of strain Q2 was sequenced using the whole genome de novo sequencing at the Illumina NovSeq platform and Pacbio Sequel platform.

\subsection{The solid fermentation of strain Q2}

On the basis of our previous research, we selected wheat grain, corn straw, and humic acid as the main material of solid fermentation medium. Orthogonal test table $\mathrm{L}_{9}\left(3^{4}\right)$ was used for studying the optimal solid-state fermentation media formula of strain Q2. The optimal solid-state fermentation media formula of strain Q2 was $66.67 \%$ wheat grain, $14.29 \%$ corn straw, $19.05 \%$ humic acid, and $65.0 \%$ moisture and the number of spores can reach $6 \times 10^{9} \mathrm{cfu} \cdot \mathrm{g}^{-1}$ (Supplementary Table S3). After drying, culture material was crushed and adjusted spores concentration containing $2 \times 10^{7} \mathrm{cfu} \cdot \mathrm{g}^{-1}$.

\subsection{Antagonism assay in vitro and in vivo}

The ability of strain Q2 to inhibit the growth of pathogenic fungi listed in Sect. 2.1 has been investigated. Antagonism of strain Q2 against pathogenic fungi was conducted in vitro using PDA plates as described in Sect. 2.3.

For the study on the control efficacy of strain Q2 in greenhouse and field, healthy bitter gourd seedlings with 2-4 leaves were selected and transplanted to health soil and pathogenic soil containing fungal pathogen inocula of Fusarium wilt, the preparation of soil infested with the pathogenic fungus was

Page $3 / 20$ 
conducted according to Wang [24]. $15 \mathrm{~g}$ of microbial agents of strain Q2 were poured for each plant in health soil (H-TP) and pathogenic soil (TP). And the equal amounts of solid fermentation medium without strain Q2 was poured for each plant health soil (Control) and pathogenic soil (S-FOM), all the seedlings were cultured at the same condition. After transplantation for 30 days, diseased incidence and disease index of bitter gourd seedlings were investigated. Control efficacy of Fusarium wilt of bitter gourd was calculated. Six classes were divided according to Chen [25]. The density of Fusarium spp. in the bitter gourd rhizosphere was estimated by metagenomic sequencing and dilution plating (using Fusarium selective medium Komada's medium[26].

\subsection{The mechanism of strain Q2 inhibiting the growth of FOM}

In order to verify the mechanism of strain Q2 inhibiting the growth of Fom. Two treatments with three replicates were set up, including the sample singlycultured of Fom (Control treatment) and the sample co-cultured with strain Q2 and Fom ( $T$ ). Spores of strain Q2 and Fom were washed with sterile water, the final concentration of $4 \times 10^{7} \mathrm{cfu} \cdot \mathrm{ml}^{-1}$ and $2.5 \times 10^{7} \mathrm{cfu} \cdot \mathrm{ml}^{-1}$, respectively. Subsequently, $0.8 \mathrm{~mL}$ of conidial suspensions of Fom combined with $0 \mathrm{ml}$ (Control), $0.05 \mathrm{ml}$ (T1), $0.5 \mathrm{ml}$ (T2), $1.0 \mathrm{ml}$ (T3), $2.5 \mathrm{ml}$ (T4), and $5.0 \mathrm{ml}$ (T5) of conidial suspensions of Q2 in multiple proportions were inoculated into $100 \mathrm{~mL}$ PDB, cocultured at $180 \mathrm{rpm} \cdot \mathrm{min}^{-1}$ and $28^{\circ} \mathrm{C}$ for $3-5$ days. The growth of Fom in different treatments was recorded by measuring the spore number of Fom. The axenic culture was filtered through three layers of gauze, and then filtrates were centrifuged at $8000 \mathrm{rpm} \cdot \mathrm{min}^{-1}$ for $10 \mathrm{~min}$, and supernatant was discarded. Spores were precipitated and then were used to analyze the effects of strain Q2 on the metabolic activity of Fom (i.e. N-acetylglucosamine content of fermentation supernatant liquid, intracellular glycerol content and malondialdehyde content, they were measured according to instructions of the ELISA kit (Jiangsu Jingmei Bioechnology Co., Ltd.)). Meanwhile, we had chosen the sample singly-cultured of Fom (Control treatment) and the sample co-cultured with strain Q2 and Fom (T3 treatment group) for transcriptome sequencing, there were named FOM and Q2-FOM, respectively.

\subsection{Identification of the role of a CFEM domain-containing protein orthologue gene from Fom}

Generation of FomCFEM deletion mutants FomCFEM deletion mutants were generated by the split-marker approach. Briefly, a 760-bp upstream sequence and a 1027-bp downstream sequence fanking the FomCFEM gene were amplified with primer pairs CFEM-AF/R and CFEM-BF/R, respectively. The hygromycin phosphotransferase $(h p h)$ gene was amplified from pCB1003 plasmid with primer pairs HYG-F/HY-R and YG-F/HYG-R. Two equal quantities of the fragments (a total of $20 \mu \mathrm{g}$ ) were transformed into protoplasts of Fom, and the resulting transformants selected on TB3 medium containing $100 \mu \mathrm{g} / \mathrm{mL}$ hygromycin B were identified by polymerase chain reaction (PCR) with primers (CFEM-F/R, CFEM-K1F/R, and CFEM-K2F/R) and southern blot analysis. For southern blot assay, genomic DNA was extracted from mycelia of each strain as described previouslyand digested with Pvu I [27, 28]. The DNA probe was amplified by PCR using the primer pairs tCFEM-F/R. Southern blot analysis was performed with a DIG High Prime DNA Labeling and Detection Starter kit I (Roche Diagnostics, Mannheim, Germany) according to the manufacturer's protocol. According to the size difference between the wild-type and $\triangle F O m C F E M$ strain hybrid fragments, the correctness of the converter was judged. The primer sequences for each gene product are listed in Supplementary Table S4.

Complementation of FomCFEM For complementation assays, a $2.6-\mathrm{kb}$ fragment bearing $1.1 \mathrm{~kb}$ of the full-length FomCFEM gene and $1.5 \mathrm{~kb}$ of its promoter was amplified with the primer pair Ech1-CF/Rand then cloned into pFL2 vector by gap repair. This fusion plasmid was reintroduced into protoplasts of the $\triangle F o m C F E M$ mutant to obtain complemented strains ( $\triangle F O M C F E M-C)$. G418-resistant transformants were screened and confirmed by PCR.

Characterization of Fom transformants To assay growth rate, the WT strain, FomCFEM deletion mutants, and FomCFEM-C transformants were cultured on PDA at $28^{\circ} \mathrm{C}$ for 5 days. Then, the colony morphology and conidia of these strains were examined after being grown on PDA plate for 7 days. For stress tolerance assay, each indicated strain was inoculated onto $\mathrm{CM}$ plates containing $0.2 \mathrm{~g} \cdot \mathrm{L}^{-1}$ Congo red (CR), $1 \mathrm{~mol} \cdot \mathrm{L}^{-1} \mathrm{sorbitol}, 0.05 \% \mathrm{SDS}, 0.7 \mathrm{~mol} \cdot \mathrm{L}^{-1} \mathrm{NaCl}$, $0.7 \mathrm{~mol} \cdot \mathrm{L}^{-1} \mathrm{KCl}$, and $5 \mathrm{mmol} \cdot \mathrm{L}^{-1} \mathrm{H}_{2} \mathrm{O}_{2}$ as previously described $[27,29]$. To analyze the conidial germination, $50 \mu \mathrm{L}$ conidial suspension $\left(1 \times 10^{5} \mathrm{conidia} \cdot \mathrm{mL}-1\right)$ of each strain was spotted on hydrophobic coverslips and incubated at $28^{\circ} \mathrm{C}$ for $12 \mathrm{~h}$. Then, the conidial germination was examined by the Nikon Eclipse $90 \mathrm{i}$ microscope (Nikon, Tokyo, Japan). Virulence assay of each strain on bitter gourd was performed as described in Sect. 2.6.

\subsection{DNA and RNA extraction and sequencing}

Metagenomic sequencing Total microbial genomic DNA from each soil sample was extracted from $0.5 \mathrm{~g}$ of soil using a FastDNA Spin kit (MP Biomedical, Santa Ana, California, USA) or DNeasyPowerSoil Kit (QIAGEN, Inc., Netherlands) according to the instruction provided by the manufacturer. The DNA quality was assessed on a NanoDrop spectrophotometer (Thermo Fisher Scientific, Waltham, MA, USA). The extracted microbial DNA was processed to construct metagenome shotgun sequencing libraries with insert sizes of 400 bp by using the Illumina TruSeq Nano DNA LT Library Preparation Kit. Each library was sequenced by the Illumina HiSeq X-ten platform (Illumina, USA) with PE150 strategy. Raw sequencing reads were processed to obtain quality-filtered reads for further analysis. Co-occurrence networks were constructed according to Zhang [30].

Transcriptome sequencing Total RNA of fungal mycelium (filtered through a filter paper) was isolated using the Trizol Reagent (Invitrogen Life Technologies), after which the concentration, quality and integrity were determined using a NanoDrop spectrophotometer (Thermo Scientific). Sequencing libraries were generated using the TruSeq RNA Sample Preparation Kit (Illumina, San Diego, CA, USA). First strand cDNA was synthesized using random oligonucleotides and SuperScript II. Second strand cDNA synthesis was subsequently performed using DNA Polymerase I and RNase H. After adenylation of the 3' ends of the DNA fragments, lllumina PE adapter oligonucleotides were ligated to prepare for hybridization. To select cDNA fragments of the preferred 200 bp in length, the library fragments were purified using the AMPure XP system (Beckman Coulter, Beverly, CA, USA). The sequencing library was then sequenced on a Hiseq platform (Illumina) by Shanghai Personal Biotechnology $\mathrm{Cp}$. Ltd. To quantify the identified gene expression in Fom, reference genome index was built by Bowtie2 (2.2.6) and the filtered reads were mapped to the Fusarium oxysporum f. sp. cubense race 1 (https://www.ncbi.nlm.nih.gov/genome/707? genome_assembly_id = 202328) reference genome using Tophat 2 (2.0.14), the default mismatch was no more than 2. We use HTSeq (0.9.1) statistics to compare the Read Count values on each gene as the original expression of the gene, and then used FPKM (fragments per kilobase of exon model per million 
mapped reads) to standardize the expression. Then we used DESeq (1.30.0) to analyze the genes of difference expression with screened conditions as follows: expression difference multiple $\mid \log _{2}$ FoldChange $>1$, significant P-value $<0.05$.

\subsection{Validation of DEGs by RT-PCR}

To confirm the transcriptome data, the genes were randomly selected and verified and their primers were designed using the Primer Premier 5.0 software listed in Supplementary Table S5. Quantitative reverse-transcription PCR (RT-PCR) was performed on a Light Cycler $\AA^{9} 96$ instrument (Roche) using SYBR Green qPCR kits (TaKaRa) according to the manufacturer's protocol. Reactions were performed at $95^{\circ} \mathrm{C}$ for $2 \mathrm{~min}, 45 \mathrm{cycles}$ of $95^{\circ} \mathrm{C}$ for $10 \mathrm{~s}, 58^{\circ} \mathrm{C}$ for $30 \mathrm{~s}$ and $72^{\circ} \mathrm{C}$ for 30 s. EF1 was used as the reference genes. The relative expression levels of the target genes were calculated using the $2^{-\triangle \triangle C T}$ approach.

\subsection{Statistical Analysis}

All these experiments were carried out using three replicates and were repeated at least twice, with reproducible results. For multiple comparisons, two-way ANOVA with post hoc LSD and Duncan were performed using the SPSS 22.0.P $<0.05$ was considered as significant.

\section{Results}

\subsection{Responses of soil physical-chemical properties and soil enzyme to the invasion of Fom}

In this study, modifications in physical-chemical properties and enzyme of the soil in which it was infected by Fom were described in Supplementary Table S6 The soil organic matter content (SOC), pH, electrical conductivity (EC), available nitrogen content (AN), available phosphorus content (AP) and available iron content (AFe) increased in S-FOM treatment. The significant increase in the value of SOC (57.7\%), pH (2.0\%), and AFe (27.7\%) were obtained in S-FOM soil. The control group had the higher values of soil fluorescein diacetate (FDA) hydrolase and polyphenol oxidase (PPO). Although the inhibitory effects of different enzymes were different, the invasion of Fom significantly inhibited these enzymes. For example, the obvious decreases in the activities of FDA hydrolase (47.5\%) and PPO (17.4\%) were obtained in S-FOM soil. Soil FDA hydrolase and PPO activity were negatively correlated with the relative abundance of $F$. oxysporum, $\mathrm{pH}$, organic matter content, and available Fe. This result indicated that the inhibition of $F$. oxysporum on enzyme activities of the soil might play critical roles in the conversion of organic matter and the change of $\mathrm{pH}$.

\subsection{Responses of community structure and diversity of soil to the invasion of Fom}

The data set including 10 samples comprised a total of 22,492,589 contigs with 2,005,895 to 2,511,389 sequences per sample (mean 2,161,810.6 (Control) and 2,336,707.2 (S-FOM)) and 21,972,887 scaffolds with 1,931,781 to 2,469,478 sequences per sample (mean 2,106,715 (Control) and 2,287,862.4 (S-FOM)). A total of 6,923 microbes were assembled. The higher observed species and Chao1 index were detected for S-FOM, whereas the difference was not significant. The Fom infected samples showed a significantly higher Simpson index and Shannon index of bacteria than Control, however, the Fom infected samples showed lower Simpson index and Shannon index of fungi than Control (Fig. 1A). When the beta diversity was assessed, the Control and S-FOM was significantly different $\left(R^{2}=0.43, P=0.013\right)$ (Supplementary Fig. S1A). Redundancy analysis (RDA) revealed that the environmental factor variable possesses significant correlation with community structure. The variable of $\mathrm{pH}\left(\mathrm{R}^{2}=0.65, P=0.017\right)$ produced significant positive correlations with $\mathrm{S}$-FOM microbes communities, the variable of FDA hydrolase $\left(\mathrm{R}^{2}=0.84, P=0.002\right)$ and PPO $\left(\mathrm{R}^{2}=0.58, P=0.043\right)$ produced significant negative correlations with S-FOM microbes communities (Supplementary Fig. S1B).

The soil was predominantly colonized by Proteobacteria, Actinobacteria, Acidobacteria, Gemmatimonadetes, Planctomycetes, Firmicutes, Nitrospirae, and Bacteroidetes, accounting for $63.0 \%$ of the total scaffolds, when the microbe was assessed at the phylum level (Fig. 1B). Ascomycota and Basidiomycota were the largest fungal taxonomic group, but accounted for a substantially lower proportion of microbe-assigned reads (Fig. 1D). The higher abundance of increased Proteobacteria, Firmicutes, and Nitrospirae were detected in the S-FOM samples that were subjected to invasion of F. oxysporum. Meanwhile, the obvious decreases in the relative abundance of Acidobacteria, Gemmatimonadetes, Planctomycetes, and Basidiomycota were obtained in S-FOM soil (Fig. 1B and D). The modifications in the composition of the core microbe at the genus level of the S-FOM samples were described in Fig. 1C, Fig. 1E, and Supplementary Fig. S1C. The invasion of Fom significantly influenced these microbes, although the influence differed in different microbes. For example, the obvious decreases in the relative abundance of Aspergillus, Pseudomonas, Sphingobium, Lysobacter, Variovorax, Sporisorium, Methylobacterium, Mycobacterium, Micromonospora, Actinoplanes, Mycolicibacterium, Amycolatopsis, Frankia, Pseudonocardia, and Ilumatobacter were obtained in S-FOM samples. Simultaneously, the relative abundance of Fusarium, Agromyces, Ramlibacter, Streptomyces, Conexibacter and Kribbella were significantly increased in S-FOM samples. Sphingomonas, Trichoderma, and Bacillus had no difference between the control sample and the S-FOM samples. At the species level, the relative abundance of Trichoderma asperellum, T. atroviride, Bacillus anthracis, B. kochii, Talaromyces stipitatus and so on showed an increased tendency in response to the invasion of F. oxysporum (Supplementary Fig. S2). Meanwhile, Fusarium sp. was composed of F. incarnatum, F. culmorum, F. delphinoides, F. dimerum, F. equiseti, F. fujikuroi, F. graminearum, F. langsethiae, F. oxysporum, F. pseudograminearum, F. solani, F. venenatum, F. verticillioides, and Unclassified Fusarium. The F. oxysporum, F. solani, and F. fujikuroi showed significantly higher proportions than others. A significant increase of the relative abundance of F. oxysporum (51.7\%) was observed in S-FOM samples, while there was no difference in other species.

\subsection{Co-occurrence networks analysis of microbial communities}

To study the effect of the invasion of Fom on microbial interactions, the networks were constructed (Supplementary Fig. S3). The co-occurrence network was constructed from 10 samples (Control and S-FOM) based on the strong and significant $(P<0.05)$ correlations of microbial species. In total, 5035 nodes ( 5025 microbial nodes and 10 environmental nodes) and 17912 edges were shared among co-occurrence networks, and the co-occurrence networks contains 89.54\%-92.13\% microbial biomass (Supplementary Table S7). The degrees of the networks followed power-law distributions, the average path length was 
significantly larger than the corresponding random networks, which indicated that co-occurrence networks was a small world (Supplementary Fig. S2 and Table S7). Meanwhile, modularity values were higher than the random networks and greater than 0.34 . This indicated that the co-occurrence networks had modular structures. For the networks, approximately $82.75 \%$ of the nodes belonged to bacteria, $7.98 \%$ of the nodes belonged to eukaryota, $7.36 \%$ of the nodes belonged to archaea, $1.91 \%$ of the nodes belonged to viruses. Proteobacteria (33.29\%), Actinobacteria (18.23\%), and Firmicutes (7.02\%) accounted for more links in the network. There are 353 modules among the networks, of which 15 modules had more than 50 nodes, such as module 1141 , module 676 , module 1458 , module 1465 , module 168 , module 1460 , module 1229 , module 1163 , module 1124 , module 1281 , module 1250 , module 1238 , module 1225 , module 1286 and module 1283 , the nodes account for $37.87 \%$ of the total number of nodes and the relative abundance of microbe the nodes account for 70.39 $75.23 \%$ of the total number of microbe, including Proteobacteria (35.26\%), Actinobacteria (20.86\%), Firmicutes (6.25\%), Euryarchaeota (2.94\%),

Thaumarchaeota (2.73\%), Ascomycota (2.47\%), and Bacteroidetes (2.15\%) (Supplementary Fig. S3 C and D). We investigated the distribution of soil microbial species by Zi-Pi plot (Supplementary Fig. S4). In the network, $94.22 \%$ of the nodes belong to the peripherals, $4.39 \%$ of the nodes belong to the connectors, $0.71 \%$ of the nodes belong to the module hubs, $0.08 \%$ of the nodes belong to the network hubs, among them, the connectors, module hubs and the network hubs were considered as key microorganisms. Among the 261 key microbial species, 159 microbial species belong to Proteobacteria, 27 microbial species belong to the Actinobacteria, and the relative abundance of 13 microbial species were increased in S-FOM samples (Table 1). The relative abundance of sp1571 (Kribbella flavida), sp1615 (Microlunatus soli), sp2002 (Streptomyces sp. 4F), sp4492 (Devosia sp.), sp5385 (Burkholderia sp.), sp5454 (Paraburkholderia sprentiae), sp5496 (Acidovorax sp.), sp5501 (Alicycliphilus denitrificans) and sp5675 (Chromobacterium vaccinii) were significantly positively correlated with the relative abundance of F. oxysporum. The relative abundance of sp5951 (Haliangium ochraceum), sp744 (Acidimicrobium ferrooxidans) and sp4354 (Bradyrhizobium erythrophlei) were negatively correlated with the relative abundance of F. oxysporum.

Table 1 The topological features of key microorganisms in co-occurrence networks and the correlation coefficient with F. oxysporum and Talaromyces purpureogenus

\begin{tabular}{|c|c|c|c|c|c|c|c|c|c|c|}
\hline \multirow[t]{2}{*}{ OTU ID } & \multirow[t]{2}{*}{ Phylum } & \multirow[t]{2}{*}{ Genus } & \multicolumn{2}{|l|}{ Relative abundance } & \multirow[t]{2}{*}{ Degree } & \multirow[t]{2}{*}{$\begin{array}{l}\text { Betw } \\
\text { centrality }\end{array}$} & \multirow[t]{2}{*}{$\mathrm{Zi}$} & \multirow[t]{2}{*}{$\mathrm{Pi}$} & \multicolumn{2}{|c|}{$\begin{array}{l}\text { Correlation } \\
\text { coefficient }\end{array}$} \\
\hline & & & Control & S-FOM & & & & & Fom & $\begin{array}{l}\text { Strain } \\
\text { Q2 }\end{array}$ \\
\hline sp1227\# & Actinobacteria & Arsenicicoccus & $1578.3 \pm 67.55$ & $1670.77 \pm 153.65$ & 6 & 3156.53 & -1.10 & 0.67 & 0.479 & 0.261 \\
\hline sp1571\# & Actinobacteria & Kribbella & $8311.72 \pm 732.74$ & $16815.91 \pm 4769.13^{\star \star}$ & 10 & 5398.30 & 1.23 & 0.64 & $0.806^{\star *}$ & 0.697 \\
\hline sp1615\# & Actinobacteria & Microlunatus & $1270.57 \pm 39.9$ & $1610.00 \pm 195.1^{\star *}$ & 8 & 638.42 & 0.37 & 0.66 & $0.794^{\star \star}$ & 0.576 \\
\hline sp2002\# & Actinobacteria & Streptomyces & $1353.06 \pm 56.39$ & $1790.82 \pm 285.96^{*}$ & 7 & 1206.15 & 0.37 & 0.69 & $0.733^{*}$ & 0.709 \\
\hline sp4492\# & Proteobacteria & Devosia & $3952.31 \pm 519.85$ & $5870.62 \pm 674.90$ ** & 5 & 1503.80 & -0.49 & 0.72 & $0.842^{\star *}$ & 0.794 \\
\hline sp4982\# & Proteobacteria & Azospirillum & $3357.51 \pm 94.17$ & $3516.69 \pm 201.64$ & 8 & 412.30 & 0.94 & 0.66 & 0.503 & 0.273 \\
\hline sp5385\# & Proteobacteria & Burkholderia & $7092.2 \pm 295.86$ & $7920.63 \pm 713.51^{*}$ & 4 & 390.17 & -1.08 & 0.63 & $0.685^{\star}$ & 0.552 \\
\hline sp5454 \# & Proteobacteria & Paraburkholderia & $1091.48 \pm 59.02$ & $1208.08 \pm 101.81$ & 3 & 5808.20 & -1.25 & 0.67 & $0.697 *$ & 0.527 \\
\hline sp5496\# & Proteobacteria & Acidovorax & $457.68 \pm 32.01$ & $617.36 \pm 56.87 \star \star$ & 3 & 337.52 & -1.57 & 0.67 & $0.939 * \star$ & 0.806 \\
\hline sp5501\# & Proteobacteria & Alicycliphilus & $2216.43 \pm 86.58$ & $2684.07 \pm 222.70$ ** & 14 & 4476.52 & -0.41 & 0.71 & $0.939 * *$ & 0.733 \\
\hline sp5675\# & Proteobacteria & Chromobacterium & $1094.98 \pm 83.71$ & $1246.80 \pm 102.88 *$ & 5 & 564.79 & -1.08 & 0.72 & $0.891^{\star *}$ & 0.709 \\
\hline sp5951 & Proteobacteria & Haliangium & $22571.04 \pm 703.57$ & $20814.08 \pm 1675.12$ & 4 & 711.62 & -1.03 & 0.63 & -0.467 & -0.491 \\
\hline sp744 $\#$ & Actinobacteria & Acidimicrobium & $1670.81 \pm 76.07^{\star \star}$ & $1539.43 \pm 91.80$ & 13 & 22530.34 & -0.30 & 0.70 & -0.600 & $-0.64 \varepsilon$ \\
\hline sp $1175^{\&}$ & Actinobacteria & Modestobacter & $4728.08 \pm 178.51$ & $4992.08 \pm 366.15$ & 10 & 1243.11 & 2.53 & 0.46 & 0.527 & 0.297 \\
\hline sp4354\& & Proteobacteria & Bradyrhizobium & $16015.93 \pm 532.84$ & $15386.41 \pm 1261.79$ & 19 & 7755.65 & 2.62 & 0.28 & -0.285 & -0.51 ! \\
\hline sp7444\& & \multicolumn{2}{|c|}{ Unclassified Bacteria } & $278853.56 \pm 5378.65$ & $292085.12 \pm 19196.42$ & 18 & 6825.71 & 2.75 & 0 & 0.455 & 0.382 \\
\hline Fom & \multicolumn{2}{|c|}{ Fusarium oxysporum } & $38.80 \pm 4.22$ & $58.23 \pm 11.67^{\star}$ & / & / & / & / & 1 & 0.794 \\
\hline $\begin{array}{l}\text { Strain } \\
\text { Q2 }\end{array}$ & \multicolumn{2}{|c|}{ Talaromyces purpureogenus } & $27.09 \pm 5.93$ & $51.87 \pm 13.13^{\star}$ & / & / & / & / & $0.794 \star \star$ & 1 \\
\hline
\end{tabular}

\# means connectorssp; \& means module hubs. * indicate a significant difference or correlation $(P<0.05)$; ** indicate a significant difference or correlation (P<0.01). 1227: Arsenicicoccus sp.; sp1571: Kribbella flavida; sp1615: Microlunatus soli; sp2002: Streptomyces sp. 4F; sp4492: Devosia sp. A16; sp4982: Azospirillum thiophilum; sp5385: Burkholderia sp.; sp5454: Paraburkholderia sprentiae, sp5496: Acidovorax sp. RAC01; sp5501: Alicycliphilus denitrificans; sp5675: Chromobacterium vaccinii, sp5951: Haliangium ochraceum; sp744: Acidimicrobium ferrooxidans; sp1175: Modestobacter marinus, sp4354: Bradyrhizobium erythrophlei; sp7444: Unclassified Bacteria. the same below 


\begin{tabular}{|c|c|c|c|c|c|c|c|c|c|}
\hline \multirow[t]{2}{*}{ OTU ID } & \multirow[t]{2}{*}{ Phylum } & \multirow[t]{2}{*}{ Genus } & \multicolumn{2}{|l|}{ Relative abundance } & \multirow[t]{2}{*}{ Degree } & \multirow{2}{*}{$\begin{array}{l}\text { Betw } \\
\text { centrality }\end{array}$} & \multirow[t]{2}{*}{$\mathrm{Zi}$} & \multirow[t]{2}{*}{$\mathrm{Pi}$} & \multirow{2}{*}{$\begin{array}{l}\text { Correlatio } \\
\text { coefficien } \\
\text { Fom }\end{array}$} \\
\hline & & & Control & S-FOM & & & & & \\
\hline sp17 & Actinobacteria & Actinobacteria & $219193.85 \pm 5453.8$ & $229361.99 \pm 17870.27$ & 48 & 17303.9 & 3.70 & 0.04 & 0.491 \\
\hline sp28 & Proteobacteria & Burkholderiales & $58742.72 \pm 4327.09$ & $75223.07 \pm 5342.81^{\star \star}$ & 15 & 2143.72 & 3.18 & 0.34 & $0.939 * *$ \\
\hline sp60 & Proteobacteria & Myxococcales & $15990.17 \pm 517.83$ & $16788.61 \pm 1396.37$ & 34 & 103336.29 & 3.93 & 0.11 & 0.588 \\
\hline sp61 & Proteobacteria & Bradyrhizobium & $16015.94 \pm 532.85$ & $15386.41 \pm 1261.79$ & 45 & 61630.75 & 3.96 & 0.13 & -0.285 \\
\hline sp78 & Proteobacteria & Anaeromyxobacter & $11863.22 \pm 432.14$ & $12436.17 \pm 999.12$ & 27 & 21087.43 & 3.02 & 0.07 & 0.552 \\
\hline sp115 & Proteobacteria & Bradyrhizobium & $7959.14 \pm 370.72$ & $7464.79 \pm 667.44$ & 44 & 21355.7 & 3.96 & 0.09 & -0.297 \\
\hline sp129 & Proteobacteria & Myxococcus & $6457.37 \pm 267.92$ & $6955.99 \pm 573.05$ & 43 & 168055.48 & 4.23 & 0.37 & 0.564 \\
\hline sp130 & Proteobacteria & Bradyrhizobium & $6510.68 \pm 226.33$ & $6357.98 \pm 519.76$ & 39 & 40592.81 & 3.37 & 0.10 & 0.006 \\
\hline sp149 & Proteobacteria & Bordetella & $5217.8 \pm 254.93$ & $6173.29 \pm 578.51 *$ & 25 & 33010.98 & 2.69 & 0.70 & $0.891^{\star \star}$ \\
\hline sp182 & Acidobacteria & Acidobacterium & $5129.82 \pm 157.81$ & $4827.77 \pm 347.97$ & 26 & 16365.47 & 2.71 & 0.14 & -0.333 \\
\hline sp192 & Proteobacteria & Thiobacillus & $4371.98 \pm 243.68$ & $5257.59 \pm 502.88^{*}$ & 21 & 7835.58 & 2.98 & 0.53 & $0.891^{\star \star}$ \\
\hline sp201 & Proteobacteria & Sinorhizobium & $4248.08 \pm 161.02$ & $4684.56 \pm 385.43$ & 43 & 444514.48 & 6.22 & 0.62 & $0.782^{\star \star}$ \\
\hline sp204 & Proteobacteria & Bradyrhizobium & $4435.02 \pm 177.85$ & $4345.54 \pm 320.6$ & 41 & 14945.07 & 3.60 & 0.09 & -0.018 \\
\hline sp214 & Acidobacteria & Terriglobus & $4440.03 \pm 75.16$ & $4250.3 \pm 283.31$ & 31 & 58495.42 & 2.86 & 0.32 & -0.333 \\
\hline sp241 & Proteobacteria & Blastochloris & $3839.65 \pm 134.9$ & $3882.32 \pm 283.18$ & 33 & 12536.46 & 2.90 & 0 & 0.236 \\
\hline sp243 & Acidobacteria & Granulicella & $4026.25 \pm 95.71$ & $3803.9 \pm 278.89$ & 34 & 61955.15 & 3.93 & 0.11 & -0.273 \\
\hline sp259 & Proteobacteria & Deltaproteobacteria & $3686.93 \pm 112.72$ & $3767.67 \pm 274.11$ & 23 & 12673.7 & 2.56 & 0 & 0.479 \\
\hline sp261 & Proteobacteria & Stenotrophomonas & $2927.55 \pm 169.54$ & $3928.56 \pm 342.72^{\star \star}$ & 14 & 6050.23 & 3.18 & 0.24 & 0.964 ** \\
\hline sp287 & Actinobacteria & Frankia & $3361.42 \pm 100.35$ & $3336.42 \pm 271.59$ & 34 & 56108.42 & 2.54 & 0.21 & 0.079 \\
\hline sp315 & Proteobacteria & Bradyrhizobium & $2977.26 \pm 114.01$ & $2986.82 \pm 221.23$ & 44 & 28845.71 & 3.60 & 0.20 & 0.115 \\
\hline sp331 & Firmicutes & Limnochorda & $2874.85 \pm 41.89$ & $2862.25 \pm 205.78$ & 26 & 18652.51 & 3.02 & 0 & 0.115 \\
\hline sp370 & Acidobacteria & Granulicella & $2525.3 \pm 114.82$ & $2422.1 \pm 191.61$ & 27 & 18444.13 & 3.17 & 0 & -0.297 \\
\hline sp372 & Acidobacteria & Acidobacteriaceae & $2524.3 \pm 69.87^{\star}$ & $2290.09 \pm 175.12$ & 30 & 34555.83 & 3.32 & 0.13 & $-0.745^{\star}$ \\
\hline sp457 & Proteobacteria & Paraburkholderia & $1709.18 \pm 67.22$ & $1883.88 \pm 175.16$ & 60 & 415485.3 & 7.83 & 0.70 & $0.758^{*}$ \\
\hline sp476 & Proteobacteria & Chelatococcus & $1682.15 \pm 24.3$ & $1704.7 \pm 132.29$ & 41 & 44814.77 & 3.60 & 0.09 & 0.358 \\
\hline sp509 & Proteobacteria & Thiodictyon & $1545.94 \pm 100.82$ & $1710.59 \pm 135.38$ & 19 & 74875.8 & 2.82 & 0.79 & 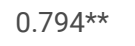 \\
\hline sp624 & Proteobacteria & Chromobacterium & $1171.53 \pm 63.5$ & $1348.25 \pm 103.31$ * & 81 & 815503.77 & 3.85 & 0.92 & $0.842^{* *}$ \\
\hline sp694 & Actinobacteria & Nocardia & $1022.74 \pm 20.87$ & $1073.01 \pm 69.13$ & 39 & 24694.67 & 2.90 & 0 & 0.624 \\
\hline sp728 & unidentified & unidentified & $1028.83 \pm 81.3$ & $978.54 \pm 80.43$ & 42 & 26429.36 & 3.20 & 0 & -0.358 \\
\hline sp852 & Proteobacteria & Ahniella & $780.82 \pm 19.17$ & $863.22 \pm 73.02$ & 17 & 105279.65 & 2.67 & 0.48 & $0.721^{\star}$ \\
\hline sp870 & Actinobacteria & unidentified & $793.18 \pm 41.23$ & $801.08 \pm 69.09$ & 43 & 17083.01 & 3.30 & 0 & 0.200 \\
\hline sp1067 & unidentified & unidentified & $550.49 \pm 39.26$ & $554.54 \pm 49.22$ & 49 & 83041.16 & 3.80 & 0.04 & 0.091 \\
\hline sp1111 & Proteobacteria & Desulfomicrobium & $544.85 \pm 29.15$ & $516.7 \pm 51.37$ & 26 & 15229.88 & 3.02 & 0 & -0.248 \\
\hline sp1120 & Proteobacteria & Desulfuromonadales & $528.33 \pm 28.83$ & $504.03 \pm 44.55$ & 27 & 53267.22 & 3.17 & 0 & -0.261 \\
\hline sp1208 & Gemmatimonadetes & unidentified & $455.52 \pm 18.75$ & $426.39 \pm 39.95$ & 29 & 15740.84 & 3.47 & 0 & -0.527 \\
\hline sp1449 & $\begin{array}{l}\text { Deinococcus- } \\
\text { Thermus }\end{array}$ & Deinococcus & $288.51 \pm 6.72$ & $283.98 \pm 20.24$ & 29 & 16332.01 & 3.32 & 0.07 & -0.079 \\
\hline sp1899 & unidentified & unidentified & $141.89 \pm 17.57$ & $125.12 \pm 19.8$ & 39 & 20524.19 & 2.90 & 0 & -0.600 \\
\hline sp5376 & Actinobacteria & Microbacterium & $6.95 \pm 2.21 *$ & $3.94 \pm 1.35$ & 23 & 90227.86 & 2.56 & 0 & $-0.867 * \star$ \\
\hline
\end{tabular}




\begin{tabular}{|c|c|c|c|c|c|c|c|c|c|}
\hline sp5882 & Ascomycota & Neurospora & $3.37 \pm 1.99$ & $4.12 \pm 1.03$ & 36 & 164.45 & 2.59 & 0 & -0.067 \\
\hline sp7622 & Crenarchaeota & Sulfolobaceae & $1.22 \pm 0.26$ & $0.96 \pm 0.59$ & 54 & 12867 & 2.55 & 0 & -0.055 \\
\hline Fom & \multicolumn{2}{|c|}{ Fusarium oxysporum } & $38.80 \pm 4.22$ & $58.23 \pm 11.67 *$ & / & / & / & / & 1 \\
\hline $\begin{array}{l}\text { Strain } \\
\text { Q2 }\end{array}$ & \multicolumn{2}{|c|}{ Talaromyces purpureogenus } & $27.09 \pm 5.93$ & $51.87 \pm 13.13^{\star}$ & / & / & / & / & $0.794^{\star *}$ \\
\hline
\end{tabular}

3.4. Isolation and screening of biocontrol agents from pathogenic soil of $\mathrm{F}$. oxysporum

The soil invaded by F. oxysporum was used for the discovery of beneficial microbial species antagonism against Fom. More than 100 microbial isolates were screened and successfully revealed 14 biocontrol agents with strong antagonistic activity to Fom on the plate, including 7 bacterial strains, 2 Penicillium sp. (1 Talaromyces sp.), and 5 Trichoderma sp.. Fom inhibition rate was 52\%-83\% (Supplementary Table S1). The secondary screening was conducted in pot trials and successfully revealed 4 biocontrol agents that have strong control effects on Fusarium wilt of bitter gourd in the greenhouse, including bacterial strain SK2 and SK6, Talaromyces sp. strain Q2, and Trichoderma sp. strain M2, their control efficacy on Fusarium wilt were 68\%-79\% (Supplementary Table S8). Meanwhile, the relative abundance of sp1571 (Kribbella flavida), sp2002 (Streptomyces sp. 4F), sp4492 (Devosia sp.), sp5496 (Acidovorax sp.), sp5501 (Alicycliphilus denitrificans), sp5675 (Chromobacterium vaccinii) and F. oxysporum were significantly positively correlated with the relative abundance of strain Q2, and the correlation coefficient between strain Q2 and F. oxysporum reaches 0.79 (Table 1).

\subsection{Micromorphology, biocontrol potential, and phylogenetic analysis of strain Q2}

Strain Q2 had strong antagonistic activity to Fom on the plate (Fig. 2A ). Colonies of strain Q2 growing moderately fast, reaching $70.46 \mathrm{~mm}$ on PDA, 49.44 $\mathrm{mm}$ on CA, $76.68 \mathrm{~mm}$ on CYA, $76.22 \mathrm{~mm}$ on $\mathrm{OA}, 70.66 \mathrm{~mm}$ on CMA, $75.32 \mathrm{~mm}$ on MM, $67.06 \mathrm{~mm}$ on SSA, $61.00 \mathrm{~mm}$ on SDA and $66.56 \mathrm{~mm}$ on SMA at $25{ }^{\circ} \mathrm{C}$ for 7 days (Fig. 2D and Supplementary Table S9). Colonies of strain Q2 on PDA moderately deep, plane; margins low, entire; marginal mycelia yellow and white; conidia pile greyish green to dull green; sometime exudates minute reddish droplets; reverse dark ruby (Fig. 2D). Under light microscope, hyphae of strain Q2 were septate, smooth to coarse, and had branched. Conidiphore of strain Q2 produces from hyphal string. Conidiophores biverticillate; stipes smooth walled, top not swollen (Fig. 2B and C), metulae three to five, divergent, 8.0-13 $\mu \mathrm{m} \times 2.3-3.0 \mu \mathrm{m}$; phialides acerose, three to six per metulae, 10-20 $\mu \mathrm{m} \times 1.8-3.0$ $\mu \mathrm{m}$; conidia oval or round, smooth walled, 3-3.5 $\times 2-2.5 \mu \mathrm{m}$. Conidia chain loose, similar cylinder shape. Ascomata not observed. The optimal growth temperature was $30^{\circ} \mathrm{C}$, maximum $55^{\circ} \mathrm{C}$; optimal growth $\mathrm{pH}$ was 7; optimal carbon source was glucose (Supplementary Fig. S5).

Strain Q2 had great potential for biological control. For example, it was able to produce $\beta-1,3-$ glucanase and chitinase (Fig. 2E and F). The $\beta-1$, 3-glucanase activity of the crude culture filtrate was $2.51 \mathrm{U} \cdot \mathrm{mL}^{-1}(\mathrm{Q} 2)$ and that of the culture filtrate of substrate induced was $3.13 \mathrm{U} \cdot \mathrm{mL}^{-1}(\mathrm{Q} 2-\mathrm{CW}), 4.20 \mathrm{U} \cdot \mathrm{mL}^{-1}(\mathrm{Q} 2-\mathrm{Lam})$ and $4.38 \mathrm{U} \cdot \mathrm{mL}^{-1}$ (Q2-CW-Lam), respectively (Fig. 2G). The chitinase activity of the crude culture filtrate was $1.79 \mathrm{U} \cdot \mathrm{mL}^{-1}(\mathrm{Q} 2)$ and that of the culture filtrate of substrate induced was $2.22 \mathrm{U} \cdot \mathrm{mL}^{-1}(\mathrm{Q} 2-\mathrm{CW}), 1.92 \mathrm{U} \cdot \mathrm{mL}^{-1}$ (Q2-cChi) and $2.40 \mathrm{U} \cdot \mathrm{mL}^{-1}$ (Q2-CW-cChi), respectively (Fig. 2H). Strain Q2 was able to inhibit mycelia growth of eleven pathogenic fungi such as F. oxysporum f. sp. momordicae (Fom), F. graminearum, F. moniliforme, F. oxysporum f. sp. cucumarinum, F. oxysporum f. sp. niveum, Pyricularia oryzae, Trichothecium roseum, Cryphonectria parasitica, Cytospora chrysosperma, Phytophthora parasitica, Rhizoctonia solani (Supplementary Table S10). After co-culture for 5 days, obvious inhibitory zone was occurred between the colony of strain Q2 and Fom, which inhibited the mycelia growth of Fom by $65.86 \%$ (Fig. 2A and Supplementary Table S10).

The DNA sequencing was used to identify the strain Q2. The lengths of nucleotide sequence for ITS, beta-tubulin gene (BenA), and calmodulin gene (CaM) was 585 bp, 446 bp and 705 bp, respectively and be submitted to GenBank with the accession number KX432212, KY047419 and KX781300. The maximum likelihood (ML) phylogenetic tree showed that strain Q2 was the same as the type strain Talaromyces purpureogenus CBS 286.36, which indicated that strain Q2 is T. purpureogenus strains (Supplementary Fig. S6A). Together with the morphological identification, we determined the strain Q2 to be T. purpureogenus, anamorphic type Penicillium purpurogenum. The genome of strain Q2 was assembled with PacBio technology, has 57 scaffolds with a length of $27.73 \mathrm{Mb}$, including 9,600 genes with the average length of 1,633 bp (Supplementary Table S11). Although, 8968 genes of strain Q2 could be annotated in the KOG database, the function of $41.1 \%$ was unknown (Supplementary Fig. S6B). Meanwhile, a total of 463 CAZymes (Carbohydrate active enzymes) have been identified, encoding 200 glycoside hydrolases (GHs), 83 glycosyl transferases (GTs), 84 carbohydrate esterases (CEs), 79 auxiliary activities (AAs), 15 carbohydrate-binding modules (CBMs), and 2 polysaccharide lyases (PLs) (Supplementary Fig. S6C). The number of CE1 (Acetyl xylan esterase), CE10 (arylesterase), GH18 (chitinase), GH3 ( $\beta$-glucosidase), GH16 (xylanase), and GH109 (a-N-acetylgalactosidase) were significantly higher than other CAZymes (Supplementary Fig. S6D).

\subsection{The control efficacy of strain Q2 on Fusarium wilt of bitter gourd}

The control efficacy of strain Q2 on Fusarium wilt of bitter gourd was 63.4\% (greenhouse) and 60.2\% (field) (Table 2). The disease incidence and disease index of bitter gourd seedlings were significantly decreased after treatment with strain Q2. Besides, the time of bitter gourd seedling infected by Fom was obviously postponed, which demonstrated that the resistance to Fusarium wilt of bitter gourd seedlings was enhanced after inoculation with strain Q2. Meanwhile, the obvious modifications the composition of the fungal communities at the genus level of the Fom pathogenic soil treated with strain Q2 were discovered in this study (Supplementary Fig. S7). The lower observed species and Chao1 index were detected in TP samples (The pathogenic soil with Fusarium inoculation was treated with antagonistic fungus T. purpureogenus strain Q2), whereas the difference was not significant, the TP samples showed a significantly lower Simpson index and Shannon index of fungi than S-FOM samples, but, the TP samples showed a significantly higher Simpson index and Shannon index of bacteria than S-FOM samples (Supplementary Fig. S7A). The relative abundance of Actinobacteria, Firmicutes, Ascomycota, Chytridiomycota, Streptomyces, Conexibacter, Sphingomonas, Rhodoplanes, Gemmatirosa, Sphingobium, Nocardioides, Mesorhizobium, Lysobacter, Mycobacterium, Micromonospora, Talaromyces, Aspergillus, Thermothelomyces, and Chaetomium were increased in TP samples. The decreases in the relative abundance of Fusarium, Cordyceps, Colletotrichum, Anthracocystis, Trichoderma, Thielavia, Cladophialophora, Gaeumannomyces, Sporisorium, Verticillium, Fonsecaea, Exophiala, Trametes, and Diplodia were observed in TP samples (Supplementary Fig. S7 B-E). Talaromyces became the largest fungal 
taxonomic group in the TP samples compared with S-FOM samples, the relative abundance of strain Q2 that obtained by comparing the genome of the strain Q2 to the metagenomic sequencing data in the TP samples was 2828.01 reads compared to S-FOM samples with 51.87 reads and control samples with 27.09 reads (Supplementary Fig. S7E and I). A random forest analysis was then applied to identify the major microbes that contribute to the variation in soil microbiota. Ascomycota, Thaumarchaeota, Euglenida, Firmicutes, Chytridiomycota, Actinobacteria, Nitrospirae, Bacteroidetes, Microsporidia and so on were the primary factor that affected features of soil of microbial community (Supplementary Fig. S7 F). Spearman's correlation network analyses confirmed that Actinobacteria, Ascomycota, Nitrospirae, Tenericutes, Bacteroidetes, Bathyarchaeota, Beckwithbacteria, Campbellbacteria, Saccharibacteria, Tectomicrobia, Woesebacteria, Wolfebacteria, Elusimicrobia, Thaumarchaeota and T. purpureogenus obviously influenced the Simpson index of soil microbiota (Supplementary Table S12). Meanwhile, T. purpureogenus seems to have a strong positive correlation with Actinobacteria, Firmicutes, Thaumarchaeota, Ascomycota, Bacillariophyta, Bacteroidetes, Beckwithbacteria, Campbellbacteria, Saccharibacteria, Woesebacteria, Wolfebacteria, Elusimicrobia, and Tenericutes (Supplementary Table S12). The relative abundance of Fusarium decreased in the TP sample (Supplementary Fig. S7G), but the number of Fusarium in the rhizosphere of bitter gourd had decreased significantly on the Fusarium selective medium (Komada's medium) (Supplementary Fig. S7H). The density of Fusarium under TP treatment was lower, with only $10,700 \mathrm{cfu} \cdot \mathrm{g}^{-1}$ soil compared to $17,600 \mathrm{cfu} \cdot \mathrm{g}^{-1}$ soil in S-FOM treatment. Meanwhile, strain Q2 could colonize in soil, but its increasing trend in soil was gradually decreasing (Supplementary Fig. S7J).

\section{Table 2 The biocontrol efficacy of strain Q2 on Fusarium wilt of bitter gourd in pot and field}

\begin{tabular}{|c|c|c|c|c|c|c|}
\hline \multirow[t]{2}{*}{ Treatment } & \multicolumn{3}{|l|}{ Pot } & \multicolumn{3}{|l|}{ Field } \\
\hline & Incidence (\%) & Disease index & Control efficacy (\%) & Incidence (\%) & Disease index & Control efficacy (\%) \\
\hline Control & I & / & / & $6.94 \pm 2.40 \mathrm{c}$ & $2.31 \pm 1.22 \mathrm{c}$ & / \\
\hline C-TP & / & / & / & $2.78 \pm 2.41 \mathrm{c}$ & $0.93 \pm 0.80 \mathrm{c}$ & / \\
\hline S-FOM & $95.70 \pm 7.45$ & $72.07 \pm 24.85$ & / & $45.56 \pm 3.85$ & $34.44 \pm 3.92$ & / \\
\hline TP & $63.20 \pm 25.19$ & $26.38 \pm 13.87$ & 63.40 & $27.78 \pm 5.09$ & $13.7 \pm 1.34$ & 60.22 \\
\hline
\end{tabular}

To constructed the co-occurrence network between Control, S-FOM and TP to explain the influence F. oxysporum f. sp. momordicae (Fom) and T. purpureogenus (Tp) to soil microbiota (Fig. 3A and 3B), we compared the differences in network-level topological features between Control, S-FOM and TP. For different treatments, soil pH was significantly lower in TP treatment than in Control and S-FOM. However, electrical conductivity (EC), available phosphorus (AP), available iron (AFe), available manganese (AMn), soil organic matter (SOC), FDA hydrolase, polyphenol oxidase (PPO), urease (UE) and acid phosphatase (ACP) were higher in TP treatment than in Control and S-FOM (Supplementary Table. S6). Similar to soil enzyme, values for the vertex number, edge number, modularity and degree centralization were significantly higher for sub-networks in TP treatment than in Control, according to Tukey HSD tests (Fig. 3C). In contrast, values for the average path length and density were lower for sub-networks in TP treatment than in Control (Fig. 3C). These results suggest that the soil microbiota in TP treatment was more closely associated than in Control. To evaluate the relative contribution of soil chemical properties and soil enzyme activity to network-level topological features of the soil microbial network. In total, compared with soil chemical properties, the soil enzyme activity contributed to more of the variation in the network-level topological features. The contribution of soil FDA hydrolase, urease (UE), acid phosphatase (ACP), soil pH, available iron (AFe) and available manganese (AMn) to the network-level topological features overwhelmed that of other environmental factors with multiple regression analysis (Fig. 3D). Compared with F. oxysporum, T. purpureogenus seems to have a stronger positive correlation with vertex number, edge number, modularity and degree centralization and negative correlation with density based on Spearman's correlation analysis (Fig. 3E). Meanwhile, $F$. oxysporum and T. purpureogenus had a strong positive correlation with SOC, AFe, S-UE and S-ACP, and T. purpureogenus also have a strong positive correlation with EC and AP (Fig. 3E). The degrees of the networks followed power-law distributions indicated that co-occurrence networks was a small world (Supplementary Fig. S8A). The co-occurrence network contained 1718 nodes (1717 microbial nodes and 1 environmental nodes), 3445 edges, and $72.73 \%-74.80 \%$ microbial biomass (Fig. 3). In the network, $98.72 \%$ of the nodes belong to the peripherals, $1.05 \%$ of the nodes belong to the connectors, and $0.17 \%$ of the nodes belong to the module hubs, according to the distribution of soil microbial species by Zi-Pi plot (Supplementary Fig. S8B). Among the 1717 key microbial species, $33.93 \%$ microbial species belong to Proteobacteria, $20.9 \%$ microbial species belong to Actinobacteria, $6.52 \%$ microbial species belong to Firmicutes, $3.78 \%$ microbial species belong to Thaumarchaeota, $3.08 \%$ microbial species belong to Euryarchaeota, and $2.91 \%$ microbial species belong to Ascomycota (Supplementary Fig. S8C). The relative abundance of Actinobacteria and Firmicutes were higher in TP treatment than in Control and S-FOM. There are 404 modules among the networks, of which 15 modules had more than 20 nodes, sunch as module 57, module 38, module 12, module 11, module 26 , module 155 , module 118 , module 130 , module 117 , module 249 , module 168 , module 162 , module 175 , module 106 and module 180 . The nodes of the 15 modules account for $41.58 \%$ of the total number of nodes. The microbial abundance of the 15 modules account for $50.09-55.50 \%$ of the total number of microbe, including Proteobacteria (36.36\%), Actinobacteria (25.17\%), Firmicutes (5.59\%), Euryarchaeota (2.94\%), Thaumarchaeota (2.94\%), Bacteroidetes (1.82\%), and Ascomycota (1.68\%). The results suggest that Proteobacteria and Actinobacteria in soil were more often located in core positions in the network than other species.

The co-occurring network was built based on S-FOM and TP, in order to more clearly observe the influence of T. purpureogenus on the network interactions among microbes. The co-occurrence network included 4954 nodes (4946 microbial nodes and 8 environmental nodes), 16275 edges, 1752 modules and 87.79\%-89.00\% microbial biomass (Supplementary Fig. S9A). Proteobacteria, Actinobacteria, Acidobacteria Planctomycetes and Firmicutes were dominant phyla (Supplementary Fig. S9B). 13 modules had more than 50 nodes, sunch as module 897, module 1725, module 1491, module 1441, module 1448, module 1468, module 1368, module 543, module 1529, module 1450, module 1409, module 1492 and module 1505 (Supplementary Fig. S9A). There are 1512 nodes, 33 phyla and 62.77\%- 64.63\% microbial biomass in 13 larger modules (Supplementary Fig. S9 C). T. purpureogenus participated in the construction of cooccurrence network as environmental factor. It showed significant negative correlations with the Acidobacteria, Firmicutes, Chloroflexi Armatimonadetes, 
Chromerida, Chytridiomycota and Chlamydiae, obvious positive correlations with Actinobacteria and Dictyoglomi (Supplementary Fig. S9 E). In contrast, F. oxysporum showed positive correlations with the Acidobacteria, Firmicutes, Chloroflexi Armatimonadetes, Chromerida, Chytridiomycota and Chlamydiae, negative correlations with Actinobacteria and Dictyoglomi. Meanwhile, T. purpureogenus tended to co-occur with Actinobacteria and Proteobacteria of module 897. Module 897 was a module with Actinobacteria as the main component (Supplementary Fig. S9 D). The microbial species, such as Kribbella flavida, Frankia casuarinae, Kitasatospora setae, Nocardiopsis alba, Streptomyces niveus and Xylanimonas cellulosilytica, in module 897 were significantly positively correlated with the relative abundance of T. purpureogenus. The results indicated that T. purpureogenus strain Q2 involved in the resistance of soil microbial communities to the invasion of F. oxysporum, and the growth and development of F. oxysporum was inhibited by T. purpureogenus and its "friends".

\subsection{Inhibition of the growth and development of Fom by strain Q2 in co-culture}

To understand the mechanisms of inhibition of growth and development of Fom by strain Q2 under natural condition. In this study, the liquid co-cultivation of strain Q2 and Fom was firstly attempted, and the growth of strain Q2 and FOM in the axenic and co-culture conditions in PDB were significantly different at 48 h. It was observed that the culture liquor of FOM appeared beige, thicker, hazy mixture, while the color of culture liquor of strain Q2 was relatively lighter and appeared red white after they were singly-cultured for two days. However, when the inoculation ratio of strain Q2 and FOM was greater than 2:1, after cocultivation for two days, the culture broth color was red and deepened with time, and the mycelia of strain Q2 were dominant (Supplementary Fig. S10). Fom hyphae from the control group was not changed under the light microscope and hyphae grown and developed normally (Fig. 4A). Strain Q2 hyphae of all the groups were normally grown and developed, and the color of strain Q2 was not changed (Fig. 4A). There were morphological changes that hypha swelling appears round, the appearance of septa digestion, cytoplasma concentration and more than globular material in hypha cells of Fom compared with control group (Fig. 4A). Propidium iodide (PI) staining showed that the Fom conidia with red pigment accumulation lost vitality in T1 to T4 samples (Fig. 4B and C). The number of conidia of Fom in culture liquor was lower in T1, T2, T3, T4 and T5 treatment, with only $8.20 \times 10^{7} \mathrm{cfu} \cdot \mathrm{mL}^{-1}, 6.73 \times 10^{7} \mathrm{cfu} \cdot \mathrm{mL}^{-1}, 9.83 \times 10^{6}$ $\mathrm{cfu} \cdot \mathrm{mL}^{-1}, 4.33 \times 10^{6} \mathrm{cfu} \cdot \mathrm{mL}^{-1}$ and $1.77 \times 10^{6} \mathrm{cfu} \cdot \mathrm{mL}^{-1}$, respectively, compared to $1.20 \times 10^{8} \mathrm{cfu} \cdot \mathrm{mL}^{-1}$ in control treatment at 5 th days of cultivation (Fig. 4D). The results showed that the hyphae growth and spores production of Fom was inhibited significantly in the co-culture by strain Q2, and the red pigment produced by Q2 penetrated cell wall and cell membrane of the dead cell of Fom and deposited.

The presence of propidium iodide and the red pigment in Fom cells indicated that the integrity of the cell wall and cell membrane of Fom were damaged. We investigated the effect of strain Q2 on malondialdehyde (MDA) concentration and triglyceride content of Fom cell. The results showed that the MDA content and triglyceride content of Fom cell increased in T1 to T4 samples compared to control (Fig. 4F and G). The MDA content and triglyceride content in T2 treatment were significantly higher than those in the control. At the same time, it is worth noting that the small fragments increased in the culture filtrate of T3 samples (Fig. 4B), and the $\mathrm{N}$-acetylglucosamine ( $\mathrm{N}$-acetylglucosamine is a hydrolysate of chitin) content in the culture filtrate of T1 to T3 samples was significantly higher than that in the control (Fig. 4E), and the content of N-acetylglucosamine gradually increases with inoculation concentration of strain Q2 increases. The results indicated that strain Q2 maybe inhibit the growth and development of Fom by destroying the integrity of the cell wall and cell membrane of Fom.

\subsection{The influence of strain Q2 on the metabolic function of Fom at transcriptional level}

A total of more than 336.47 million high quality clean reads were obtained after removing duplicate reads, trimming adapters and low quality sequences from six libraries. Q30 percentage was over $92 \%$ for all the samples. These data showed that the RNA-Seq quality was applicable for further analysis. In FOM samples, $89.20 \%$ to $89.73 \%$ of the total reads were mapped to the genome of $F$. oxysporum $\mathrm{f}$. sp. cubense race 1 ; and more than $95 \%$ of the reads in each sample were uniquely mapped to the genome. In Q2-FOM samples, $28.25 \%$ to $47.17 \%$ of the total reads were mapped to the genome of $F$. oxysporum f. sp. cubense race 1 ; and $96.86 \%$ to $97.15 \%$ of the reads in each sample were uniquely mapped to the genome (Supplementary Table S13).

Differentially expressed genes (DEGs) were identified between FOM and Q2-FOM groups through comparing the expected read counts normalized using the DEseq package. To compare the expression levels of the DEGs between two groups, sequences count based differential expression analysis was performed. It was observed that 4,893 genes expression levels in FOM were identified as differentially expressed in FOM and Q2-FOM groups, including 2,435 up-regulated and 2,458 down-regulated (Supplementary Fig. S11A). The Gene Ontology analysis (GO) and KEGG analysis of DEGs was further conducted (Supplementary Fig. S11B and C). Following GO annotation, the DEGs were classified into different groups belonging to biological process (BP), molecular functions (MF) and cellular component (CC) categories. In the all category, the highly represented groups were 'intrinsic component of membrane' (410 up-regulated genes and 748 down-regulated genes), 'integral component of membrane' (410 up-regulated genes and 744 down-regulated genes), 'membrane part' (416 up-regulated genes and 748 down-regulated genes), 'membrane' (428 up-regulated genes and 762 down-regulated genes), 'oxidoreductase activity' (355 up-regulated genes and 207 down-regulated genes), 'transmembrane transport' (149 up-regulated genes and 256 down-regulated genes) and 'transmembrane transporter activity' (65 up-regulated genes and 146 down-regulated genes) groups. In the Q2-FOM groups, the genes associated with membrane and transport appeared to be the most significantly enriched. To further investigate the biochemical pathways of these DEGs in the process of T. purpureogenus inhibiting the growth of Fom, we mapped all DEGs identified in the RNA sequencing to terms in the KEGG database. The results showed the DEGs could be classified into four pathways, including metabolism, genetic information processing, environmental information processing and cellular processes. Top 20 enriched KEGG pathways associated with DEGs affected by T. purpureogenus were showed in Supplementary Fig. S11B, including glyoxylate and dicarboxylate metabolism, glutathione metabolism, valine, leucine and isoleucine degradation, glycerophospholipid metabolism, phenylalanine metabolism, nitrogen metabolism, propanoate metabolism, pyruvate metabolism, ABC transporters, galactose metabolism, tyrosine metabolism and glycerolipid metabolism. These results further indicated that stress of strain Q2 may greatly influence carbohydrate metabolism, amino acid metabolism, lipid metabolism, energy metabolism, component of membrane and membrane transport of F. oxysporum.

Based on the STRING output, the interaction of 371 coding genes was described (Fig. 5A), including cell cycle relating genes, starch and sucrose metabolism relating genes, amino sugar and nucleotide sugar metabolism relating genes, glycan biosynthesis and metabolism relating genes and biosynthesis of secondary metabolites relating genes (Supplementary Table S14). Real-time PCR was used to validate the expression levels of thirteen genes (Fig. 5C),

Page $10 / 20$ 
including cell cycle relating genes, starch and sucrose metabolism relating genes, amino sugar and nucleotide sugar metabolism relating genes and glycan biosynthesis and metabolism relating genes. Among these genes, FOC1 g10015395 (CDC5, Cell cycle serine/threonine-protein kinase), F0C1 g10015375 (CG21, G2/mitotic-specific cyclin-B), FOC1 g10014984 (MPG1, Mannose-1-phosphate guanyltransferase), FOC1 g10014983 (HXKG, Glucokinase), FOC1 g10014811(GNPI1, Glucosamine-6-phosphate isomerase 1), FOC1 g10014809 (NAGA, N-acetylglucosamine-6- phosphate deacetylase), FOC1 g10014793 (INV, Invertase), FOC1 g10014440 (GPI1, N-acetylglucosaminyl- phosphatidylinositol biosynthetic protein), FOC1 g10013913 (ARK1, Serine/threonine-protein kinase), FOC1 g10013617 (BGL1B, Beta-glucosidase 1B), FOC1 g10012541 (TREA, Trehalase), FOC1 g10012205 (MALT, Alpha-glucosidase), FOC1 g10012008 (GPI18, GPI mannosyltransferase 2), FOC1 g10010259 (BIR1, Protein), FOC1 g10009667 (GPI15, Phosphatidylinositol N-acetylglucosaminyltransferase subunit), FOC1 g10009617 (WSC1, cell wall integrity and stress response component 1), FOC1 g10009158 (TPSY, Putative alpha, alpha-trehalose-phosphate synthase), FOC1 g10008820 (AGDC, Probable alpha/beta-glucosidase), FOC1 g10008111 (ASE1, Anaphase spindle elongation protein 1), FOC1 g10006897 (GPI2, Phosphatidylinositol N-acetylglucosaminyltransferase subunit), FOC1 g10005945 (TPS2, Trehalose-phosphatase), FOC1 g10005528 (CHS7, Chitin synthase 7), FOC1 g10003572 (AMYG, Glucoamylase) and FOC1 g10003114 (BUB1, Checkpoint serine/threonine-protein kinase) were down-regulated in Q2FOM. FOC1 g10015825 (GUN, Endoglucanase), FOC1 g10015543 (AGM1, Phosphoacetylglucosamine mutase), FOC1 g10012466 (GAL10, Bifunctional protein) and FOC1 g10011050 (YBB2, Probable mannose-1-phosphate guanyltransferase) were up-regulated in Q2-FOM. Four GPI biosynthesis and one chitin synthases genes were all down-regulated in Q2-FOM. The gene expression results from the real-time PCR were consistent with the transcriptome data (Fig. 5B).

We found a gene with CFEM domains (the characteristic eight cysteine residues at N terminus) and putative GPI-anchored site (FOC1 g10014283) in further analysis of differentially expressed genes. The expression level of FOC1 g10014283 was significantly decreased in Q2-FOM samples compared to FOM samples, although his function is unknown in F. oxysporum (Fig. 6A and Supplementary Table S14). We experimentally isolated and characterized an FOC1 g10014283 orthologue, FomCFEM, in F. oxysporum f. sp. momordicae.

\subsection{FomCFEM is important for full virulence and stress tolerance}

The SMART-PFAM revealed that FomCFEM consists of a CFEM domain, a signal peptide, and the potential GPI-modification site (the amino acids $\mathrm{N}^{291}$ and $\mathrm{A}^{292}$ ) (Supplementary Fig. S12A and Supplementary Fig. S13). A phylogenetic analysis of the putative FomCFEM, homologous sequences of Fusarium and other fungal genes with CFEM domain indicated that homologous sequences of FomCFEM were common in Fusarium and the FomCFEM is closely homologous to the gene with CFEM domain from Trichoderma asperellum (GFP54046.1) (Supplementary Fig. S12B). To assess functions of FomCFEM, in $F$. oxysporum f. sp. momordicae by homologous recombination, FomCFEM deletion transformants harboring a hygromycin-resistance gene instead of the entire FomCFEM were generated. After PCR and Southern blot assays, the predicted FomCFEM separate mutants were obtained (Supplementary Fig. S12C and Fig. $\mathrm{S} 14 \mathrm{~B}$ ). One of the mutants (designated as $\triangle$ FomCFEM) was selected for complementation by reintroducing the entire FomCFEM gene under the control of its native promoter into the $\triangle$ FomCFEM mutant (Supplementary Fig. S12D).

To determine the role of FomCFEM in pathogenicity, the rhizosphere soil of bitter gourd seedlings were inoculated with conidial suspensions of WT, $\triangle F o m C F E M$ mutant, and $\triangle F$ FomCFEM-C strains (the inoculation amount was $1 \times 10^{6} \mathrm{spores} / \mathrm{g} \cdot \mathrm{soil}$ ). The disease incidence and disease index of bitter gourd seedlings were significantly decreased after treatment with $\triangle$ FomCFEM mutant (Fig. 6B and C). The $\triangle$ FomCFEM mutant resulted in a $58.8 \%$ reduction in virulence compared to the WT strain. These results suggest that FomCFEM is essential for full virulence in F. oxysporum $\mathrm{f}$. sp. momordicae. Although conidial production of $\triangle$ FomCFEM was decreased compared with WT and $\triangle F O M C F E M-C$ strains, there was no difference in the conidium morphology and conidial germination of $\triangle$ FomCFEM, WT, and $\triangle$ FomCFEM-C (Fig. 6F-G). In addition, to investigate whether FomCFEM is involved in external stress tolerance, growth assays of indicated strains on $\mathrm{CM}$ media supplemented with salt stress $\left(0.7 \mathrm{~mol} \cdot \mathrm{L}^{-1} \mathrm{NaCl}\right.$ and $\left.0.7 \mathrm{~mol} \cdot \mathrm{L}^{-1} \mathrm{KCl}\right)$, osmotic stress $\left(1 \mathrm{~mol} \cdot \mathrm{L}^{-1}\right.$ sorbitol), $\mathrm{H}_{2} \mathrm{O}_{2}(5$ $\left.\mathrm{mmol} \cdot \mathrm{L}^{-1}\right)$ and cell wall stress $\left(0.05 \% \mathrm{SDS}\right.$, and $\left.0.2 \mathrm{~g} \cdot \mathrm{mL}^{-1} \mathrm{CR}\right)$ were analyzed, respectively. The results showed that the $\triangle$ FomCFEM strain was more hypersensitive to the cell wall stress compared with WT and $\triangle F O m C F E M-C$ strain (Fig. 6D and E). These results indicated that $\triangle F O m C F E M$ may play an important role in the integrity and function of the cell wall of $F$. oxysporum f. sp. momordicae.

\section{Discussion}

Soil microbiota, including microbial biomass, communities, diversity and the abundance of specific microbial groups, is seen to be critical for the maintenance of soil health, soil quality, and soil enzyme activities [6,31]. If the soil microorganisms are compared as the genetic material of the soil, each microbe is like the functional genes, and they are associated and interact with each other. However, pathogens of soil-borne disease, like cancer genes, seriously destroy microbical network and the health of the soil. The invasion of pathogens such as Ralstonia solanacearum and F. oxysporum would trigger drastic changes in the microbiome composition leading to the decline in disease suppression and soil quality represented by an increase in plant pathogens and loss of microbial diversity and community functioning, and once-invaded soil microbial communities could become more susceptible to secondary invasions during following crops seasons [32,33,34]. In our study, the implications of the exogenous F. oxysporum on the microbiome were primarily reflected by the changing pathogen of Fusarium wilt, microbial abundance, and microbial diversity. While the invasion of exogenous $F$. oxysporum increased fungal abundance and bacterial diversity, the relative abundance of the microbial species showed a different change. The previous investigations also reported that fungal diversity differed significantly between the suppressive and conducive soils, high soil fungal richness significantly correlated to a higher Fusarium wilt [5, 32]. The relative abundance of $F$. oxysporum $\mathrm{f}$. sp. cubense, fungal or bacterial richness and diversity were higher in the diseased soils than in the disease-free soils [34]. It has been proved that pathogens were strongly restricted in growth by the activities of specific rhizosphere microorganisms. Oxalobacteraceae, Burkholderiaceae, Sphingobacteriaceae, and Sphingomonadaceae were significantly more abundant in the rhizosphere upon the invasion of $R$. solani in disease-suppressive soils [35]. Suppressive soils provide the best examples of natural microbe-based defense the invasion of pathogens, whereby via the soil microorganisms build up the first line of defense against soilborne pathogens. However, it is unknown what kind of army that built up by soil microorganisms defense against soilborne pathogens when the inoculum of a soilborne pathogen is added to an unamended or raw field soil. We attempted to understand how indigenous microbiomes can reduce the occurrence of Fusarium wilt, even in the conducive soils. When it was assessed in more detail, we found that the

Page $11 / 20$ 
invasion of $F$. oxysporum significantly oppressed the growth of the dominant microbes and activated the growth of non-dominant microbes. The relative abundance of Gemmatimonadetes, Bradyrhizobium, Luteitalea, Conexibacter, Rhodoplanes, Cordyceps, Gemmatirosa, Aspergillus, and Thielavia decreased in S-FOM samples, while the relative abundance of many non-dominant microbes increased in S-FOM samples, such as Kribbella sp., Devosia sp., Alicycliphilus sp., Chromobacterium sp., Talaromyces sp., and Streptomyces sp.(dominant microbes). The relative abundance of Trichoderma and Bacillus that have been widely used as a biological control agent against various pathogens were no difference between control samples and S-FOM samples [15, 16, 17, 18]. But, the relative abundance of $T$. asperellum, $T$. atroviride, B. anthracis, B. kochii, and so on showed an increased tendency in response to the invasion of $F$. oxysporum. Microbes response to invasive $F$. oxysporum, and these population dynamics have diverse consequences for microbial fitness, microbial community structure, and soil functional. The interactive patterns within microbes and F. oxysporum may be positive (mutualism, synergism, or commensalism), negative (predation, parasitism, antagonism, or competition), or neutra. We attempted to shed light on the interactive relevance between the variable tendency of microbes and F. oxysporum, and found the biocontrol agent from these changed indigenous microorganisms, although it is unknown that the role of these microorganisms plays in the defense against the invasion of Fusarium. We speculated that these microbes with an increase tendency may be associated with suppression of Fusarium wilt.

Biological control has gained a great contribution to control Fusarium wilt in the last years [36]. A large number of biological control agents (BCAs) with variable effectiveness against $F$. oxysporum have been applied [37, 38], and many of them reached the stage of field trials [39]. But most biocontrol fungi are still in the theoretical or conceptual stage owing to several factors including stability in field and difficulty in solid fermentation. Obtaining these biocontrol fungi with stable biocontrol effect and easy fermentation are the main factor for their successful application. In our study, we obtained 14 biocontrol agents with strong antagonistic activity to F. oxysporum on plate, originally separated from the pathogenic soil of Fusarium wilt. In the comprehensive evaluation of the 14 biocontrol agents, we found T. purpureogenus strain Q2 had more stable and constant biocontrol efficacy on Fusarium wilt than Trichoderma and Bacillus in the greenhouse and field and the strain Q2 could stably colonize in the soil. Meanwhile, the strain Q2 showed the biocontrol potential against various pathogenic fungi, especially F. oxysporum. However, the biocontrol strains (bacteria or fungi) with an antagonistic effect on pathogen propagule initiate sustained disease suppression after reaching threshold population sizes ( $10^{5} \mathrm{cfu} / \mathrm{g}$ ) [40]. In our study, the Fusarium wilt still occurred, although the relative abundance of strain Q2 increased in the process of $F$. oxysporum invading the soil, this does not prove that the total microbiome including strain Q2 did not contribute to reduce the incidence of Fusarium wilt. The strain Q2 reached the threshold of population sizes $\left(1 \times 10^{6} \mathrm{cfu} / \mathrm{g}\right)$ by human intervention and started continuous disease suppression through inhibiting the growth and development of $F$. oxysporum. This has also been observed during the co-culture of strain Q2 and F. oxysporumf. sp. momordicae. Meanwhile, the strain Q2 can also excite the activity of other beneficial microorganisms such as actinomycetes. These results show that strain Q2 directly or indirectly suppresses the growth of $F$. oxysporum in the soil, and strain Q2 may be just the key player, other microbes play a supporting role.

Talaromyces species have a wide range of applications and are often used to produce enzymes and pigments in agriculture and industry. Talaromyces sp. has a higher number of proteins with carbohydrate-active enzyme (CAZymes) than other sequenced Penicillium based on sequencing of genome[41-43]. This indicates that Talaromyces sp. has stronger biological potential than Penicillium sp.. Talaromyces sp. as a potential biological control agent against fungal plant pathogen can be traced back to 1989 [44]. T. flavus isolates from the ginseng seed was very effective applications in improving dehiscence and germination and showed antagonistic activities against fungal plant pathogens, such as Cylindrocarpon destructans, Rhizoctonia solani, Sclerotinia nivalis, Botrytis cinerea, and Phytophthora capsici[45]. In our work, we found strain Q2 can grow on a variety of nitrogen source such as beef extract, carbamide, peptone, ammonium chloride, ammonium sulfate and ammonium nitrate, and carbon source such as glucose, starch, lactose, maltose, sucrose, D-furctose. Strain Q2 also have high tolerance to acid and temperature conditions, was able to soundly grow under the conditions of temperature ranged from 15 to $45^{\circ} \mathrm{C}$ and $\mathrm{pH} 4-10$. Meanwhile, strain Q2 has a higher number of CAZymes than other sequenced $P$. purpurogenum MYA-38 and can secrete the cell wall-degrading enzymes including $\beta-1$, 3-glucanase and chitinase [41]. Strain Q2 could produce more $\beta$-1, 3-glucanase and chitinase in culture medium containing cell wall fragments of $F$. oxysporum. Digestion of the fungal cell wall by microbial chitinase, chitosanase, and $\beta$-1, 3-glucanase may be one of mechanisms for biocontrol of soil-borne plant pathogens [17, 46-48]. Two strains of Clostridium beijerinckii can produce the cell wall-degrading enzymes and have the ability to kill F. oxysporum f. sp. spinaciae [48]. To understand the interaction of the strain Q2 and Fom, we initially conducted a co-cultivation with strain Q2 and Fom. The result showed that growth of Fom was significantly inhibited in the presence of strain Q2. In addition, $\beta$-1, 3-glucanase and chitinase enzymes production was improved in a combination inoculated with strain Q2 and Fom. Similarly, Copete-Pertuz identified a triple combination inoculated with Trichoderma viride and Aspergillus sterreus into a 7th day pre-culture of Leptosphaerulina sp. improved ligninolytic enzymes production when compared to the Leptosphaerulina sp. monoculture [49]. Meanwhile, our results showed that the content of malondialdehyde (MDA) and chitin of $F$. oxysporum mycelia had changed under the presence of strain Q2. This indicated that the change of the membrane permeability and cell wall structure of $F$. oxysporum hypae, and the damage degree of membrane system and cell wall was increased with the increase of strain Q2 density. The study on transcriptomes also brought useful information on the interaction between strain Q2 and Fom. RNA-seq data indicated that $F$. oxysporum membrane components, as well as transmembrane transport, seemed to have been seriously affected by the strain Q2 at the transcriptome level. The inhibitions observed on both growth and membrane function of Fom in our study were in accordance with those of Strub [50]. The membrane components and transmembrane transporter of $F$. verticillioides were affected by Streptomyces sp. AV05 during their co-cultivation [50].

In the model fungus Saccharomyces cerevisiae, the cell wall and cell membrane play an important role that it's defining cell shape during growth, mating, sporulation; and presents adhesive glycoproteins to other yeast cells [51, 52]. In the fungal plant pathogen $F$. oxysporum, the integrity of the cell wall and cell membrane not only plays an important role in mycelium growth but also associated with plant interaction [53]. In our study, we discovered that several pathways associated with cell wall morphogenesis, such as starch and sucrose metabolism, amino sugar and nucleotide sugar metabolism and GPI biosynthesis were drastically influenced by strain Q2. In the further analysis of the transcriptional profiling, large numbers of genes associated with Fom cell integrity were down-regulation, including the cell wall relating genes and cell membrane relating genes, have been proved to play an important role in the fungal growth and pathogenicity [54]. In this work, the expression level of cell wall and cell membrane relating genes decreased significantly, such as $C H S 1$, CHS2, CHS3, CHS7, YEA4, GPI1, GPI2, GPI15, GPI12, SMP3 and GPI18. CHS7 is a limiting factor in the export of CHS3, CHS7 deletion leads to CHS3 aggregates

Page $12 / 20$ 
in the ER and reduces chitin synthase III (CSIII) activity [55, 56]. YEA4 is the second ER membrane protein with UDP-GlcNAc transport activity, whose transport activity contributes to chitin synthesis [57]. GPI-anchored proteins, which anchored to the outer layer of the plasma membrane through a C-terminal GPI

anchor, are essential for growth, viability, morphogenesis, signaling transmission, reproduction and disease pathogenesis in all eukaryotic cells [58-60]. GPI1, GPI15, and GPI2 are core subunits of GPI-GIcNAc transferase complex, initiate the GPI biosynthesis. SMP3 and GPI18 are involved in GPI mannosylation. GPI12 catalyzes N-acetylglucosaminyl phosphatidylinositol de-N-acetylated, form glucosaminyl phosphatidylinositol. Once the GPI biosynthesis is blocked, the growth and development of the fungus will be affected. Meanwhile, multiple GPI-modified wall proteins of the fungus were important to osmotic stability and mortality [29]. In this study, we isolated and characterized a CFEM protein FomCFEM (homologous sequences of FOC1 g10014283) with a putative GPI modification site from F. oxysporum f. sp. momordicae. Nevertheless, the specific roles of the FomCFEM in F. oxysporum remain largely unknown. According to the results which we had obtained, the FomCFEM deletion mutant strain was more hypersensitive to the cell wall and stress tolerance compared with WT and $\triangle F O M C F E M-C$ strains, indicating that FomCFEM positively regulate the tolerance of F. oxysporum f. sp. momordicae to osmotic stabilizers. Meanwhile, the FomCFEM plays an important role in the full virulence of $F$. oxysporum $\mathrm{f}$. sp. momordicae. Similar result had also been reported in other studies that the gene with CFEM domain and putative GPI-anchored site involved in virulence, conidiation and stress tolerance of Botrytis cinerea [29]. Therefore, strain Q2 suppresses the expression level of FomCFEM, which may be play an important role in the prevention and control of Fusarium wilt.

T. purpureogenus strain Q2 was a soil and rhizosphere microorganisms, isolated from the rhizosphere of healthy plant that planted in the pathogenic soil of F.oxysporum. In the present study, strain Q2 has antagonistic activity against a variety of pathogens, especially against $F$. oxysporum and has a good control effect on Fusarium wilt of bitter gourd. Our results indicated that the mechanisms of strains Q2 protected bitter gourd was to reduce the relative abundance of rhizospheric F.oxysporum by direct and indirect inhibiting the growth and development of $F$. oxysporum, because the positive correlations were found between the disease incidence and growth degree of F. oxysporum [61]. In summary, strain Q2 was a good strain with the potential for biological control of Fusarium wilt. This work could provide a new potential biocontrol agent for the biocontrol of Fusarium wilt and soil-born diseases of other horticultural crops.

\section{Conclusions}

Our study demonstrates that we have screened a efficient biocontrol agents, Talaromyces purpurogenus strain Q2, from microorganisms with resistance to the invasion of F. oxysporum. The biocontrol mechanisms of T. purpurogenus strain Q2 protected bitter gourd from F. oxysporum is to reduce the relative abundance of rhizospheric F. oxysporum by inhibiting the growth and development of $F$. oxysporum. This work can provide a new biocontrol agent for biocontrol of Fusarium wilt and soil-born diseases of other horticultural crops.

\section{Abbreviations}

PDA: Potato Dextrose Agar

CFU: Colony Forming Units

DNA: Deoxyribonucleic Acid

RNA: Ribonucleic Acid

PCoA: Principal Coordinate Analysis

PCR: Polymerase Chain Reaction

RT-PCR Reverse: Transcription-Polymerase Chain Reaction

Vertex number: Number of classes

Edge number: Number of potential links between associated classes

Average path length: The average network distance between all pairs of nodes

Density: The intensity of connections among nodes

Modularity: Scores weighted the number of a graph division into sub-graphs

Degree centralization: Creating a graph level centralization measure from the centrality scores of the nodes

\section{Declarations}

Ethics approval and consent to participateNot applicable

Consent for publicationNot applicable

Availability of data and materialThe raw Illumine sequence data of metagenomic data have been deposited in the sequence read archive (SRA submission: SUB9063041) at NCBI under Bioproject accession \#PRJNA701786. The raw Illumine sequence data of RNA-seq are available (SRA accession: SRR10416247SRR10416252) in the NCBI under Bioproject accession \#PRJNA588316. The genome sequence data of Talaromyces purpureogenus strain Q2 is also available (Genomes submission: JABUIS000000000) in the NCBI under Bioproject accession \#PRJNA635544. Please contact author for data requests.

Page $13 / 20$ 
Competing interestsThe authors declare that they have no competing interests.

FundingThis research was supported by Special Fund for Agro-Scientific Research in the Public Interest of China (201503110-12); The Provincial Key Research and Development Program of Shandong (2019JZZY010714) and The Major Projects \& Key Research Program for Conversion from Old to New Kinetic Energy of Shandong (2019XJDNZH-08-38-01).

Authors' contributionsYehanTian performed all experiments; Yehan Tian and Kexiang Gao designed the study. Yehan Tian analyzed the data and generated the first draft of the manuscript; Kexiang Gao edited and revised the manuscript; All authors read and approved the final manuscript.

AcknowledgementsThe authors thank Prof. Yucan Zhang for a gift of seeds of bitter gourd.

\section{References}

1. Xiao CH, Yu XM, He YY, Deng XZ, Tang E. Studies on the biological characteristics of the pathogen of cucurbit wilt. Plant Protection. 2008; $34(2)$, 83-86.

2. Kashiwa T, Kozaki T, Ishii K, Turgeon BG, Teraoka T, Komatsu K, Arie T. Sequencing of individual chromosomes of plant pathogenic Fusarium oxysporum. Fungal Genetics and Biology. 2017; 98, 46-51.

3. Arie T. Phylogeny and phytopathogenicity mechanisms of soilborne Fusarium oxysporum. Journal of General Plant Pathology. $2010 ; 76$ (6), $403-405$.

4. Gordon TR. Fusarium oxysporum and the Fusarium wilt syndrome. Annual Review of Phytopathology. 2017; 55, $23-39$.

5. Shen ZZ, Penton CR, Lv NN, Xue C, Yuan XF, Ruan YZ, Li R, Shen QR. Banana Fusarium wilt disease incidence is influenced by shifts of soil microbial communities under different monoculture spans. Microbial Ecology. 2017; 75(3), 739-750.

6. Zhao S, Chen X, Deng S, Dong X, Song A, Yao J, Chen F. The effects of fungicide, soil fumigant, bio-organic fertilizer and their combined application on chrysanthemum Fusarium wilt controlling, soil enzyme activities and microbial properties. Molecules. 2016; $21(4), 526$.

7. McKeen CD, Wensley RN. Longevity of Fusarium oxysporum in soil tube culture. Science. 1961; 134(3489), 1528-1529.

8. Kaur R, Kaur J, Singh R. Nonpathogenic Fusarium as a biological control agent. Plant Pathology Journal. 2010; 61(10), $1213-1217$.

9. Huang XQ, Cai ZC. Soil microbes and control of soil-borne diseases. Bulletin of Chinese Academy of Sciences. 2017; 32(6), 593-600.

10. Dita M, Barquero M, Heck D, Mizubuti E, Staver CP. Fusarium wilt of banana. current knowledge on epidemiology and research needs toward sustainable disease management. Frontiers in Plant Science. 2018; 9, 1468.

11. Li C, Chen S, Zuo C, Sun Q, Ye Q, Yi G. The use of GFP-transformed isolates to study infection of banana with Fusarium oxysporum f. sp. cubense race 4 . European Journal of Plant Pathology. 2011; 131(2), 327-340.

12. Srinivas C, Nirmala Devi D, Narasimha Murthy K, Mohan CD, Lakshmeesha TR, Singh B, Chandra Nayaka S. Fusarium oxysporum f. sp. lycopersici causal agent of vascular wilt disease of tomato. biology to diversity- A review. Saudi Journal of Biological Sciences. 2019; 26(7), $1315-1324$.

13. Li N, Alfiky A, Wang W, Islam M, Nourollahi K, Liu X, Kang S. Volatile compound-mediated recognition and inhibition between Trichoderma biocontrol agents and Fusarium oxysporum. Frontiers in Microbiology. 2018; 9, 2614.

14. Passera A, Vacchini V, Cocetta G, Shahzad GI, Arpanahi AA, Casati P, Ferrante A, Piazza L. Towards nutrition-sensitive agriculture. an evaluation of biocontrol effects, nutritional value, and ecological impact of bacterial inoculants. The Science of the Total Environment. 2020; $724,138127$.

15. Castillo AG, Puig CG, Cumagun C. Non-synergistic effect of Trichoderma harzianum and Glomus spp. in reducing infection of Fusarium wilt in banana. Pathogens. 2019; 8(2), 43.

16. Kim JD, Han JW, Lee SC, Lee D, Kim BS. Disease control effect of strevertenes produced by Streptomyces psammoticus against tomato Fusarium wilt. Journal of Agricultural and Food Chemistry. 2011; 59(5), 1893-1899.

17. Abbasi S, Safaie N, Sadeghi A, Shamsbakhsh M. Streptomyces strains induce resistance to Fusarium oxysporum f. sp. lycopersici race 3 in tomato through different molecular mechanisms. Frontiers in Microbiology. 2019; 10, 1505.

18. Jiang CH, Yao XF, Mi DD, Li ZJ, Yang BY, Zheng Y, Guo JH. Comparative transcriptome analysis reveals the biocontrol mechanism of Bacillus velezensis F21 against Fusarium wilt on watermelon. Frontiers in Microbiology. 2019; 10, 652.

19. Visagie CM, Yilmaz N, Frisvad JC, Houbraken J, Seifert KA, Samson RA. Five new Talaromyces species with ampulliform-like phialides and globose rough walled conidia resembling T. verruculosus. Mycoscience. 2015; 56(5), 486-502.

20. Su L, Niu YC. Multilocus phylogenetic analysis of Talaromyces species isolated from cucurbit plants in China and description of two new species, $T$. cucurbitiradicus and T. endophyticus. Mycologia. 2018; 110(2), 375-386.

21. White TJ, Bruns T, Lee S, Taylor J. Amplification and direct sequencing of fungal ribosomal RNA genes for phylogenetic. New York: Academic Press. 1990; p 315-322.

22. Glass NL, Donaldson GC. Development of primer sets designed for use with the PCR to amplify conserved genes from filamentous ascomycetes. Applied and Environmental Microbiology. 1995; 61(4), 1323-1330.

23. Wang B, Wang L. Penicillium kongii, a new terverticillate species isolated from plant leaves in China. Mycologia. 2013; 105(6), 1547-1554.

24. Wang YF, Tian YH, Wei YJ, Gao KX. Screening of optimum combination of biocontrol bacteriathymexazol and studies on its control effect on bitter gourd Fusarium wilt. China Vegetables. 2019; $7.67-73$

25. Chen ZD, Huang RK, Li QQ, Wen JL, Yuan GQ. Development of pathogenicity and AFLP to characterize Fusarium oxysporum f. sp. momordicae isolates from bitter gourd in China. Journal of Phytopathology. 2015; 163, 202-211. 
26. Castaño R囚Borrero $C \otimes T$ Trillas MI\Avilés M. Selection of biological control agents against tomato Fusarium wilt and evaluation in greenhouse conditions of two selected agents in three growing media. Journal of the International Organization for Biological Control. 2013; 58.105-116.

27. Tang L, Yu X, Zhang L, Zhang L, Chen L, Zou S, Liang Y, Yu J, Dong H. Mitochondrial FgEch1 is responsible for conidiation and full virulence in Fusariumgraminearum. Current genetics. 2020; 66(2), 361-371.

28. Leslie JF, Summerell BA. The Fusarium laboratory manual. Blackwell Pub Professional, Hoboken, 2006

29. Zhu W, Wei W, Wu Y, Zhou Y, Peng F, Zhang S, Chen P, Xu X. BcCFEM1, a CFEM domain-containing protein with putative GPI-anchored site, is involved in pathogenicity, conidial production, and stress tolerance in Botrytis cinerea. Frontiers in microbiology. 2017; 8, 1807.

30. Zhang BG, Zhang J, Liu Y, Shi P, Wei GH. Co-occurrence patterns of soybean rhizosphere microbiome at a continental scale. Soil Biology \& Biochemistry. 2018; 118, 178-186.

31. Bonilla N, Gutiérrez-Barranquero JA, Vicente AD, Cazorla FM. Enhancing soil quality and plant health through suppressive organic amendments. Diversity. $2012 ; 4,475-491$

32. Siegel-Hertz K, Edel-Hermann V, Chapelle E, Terrat S, Raaijmakers J M, Steinberg C. Comparative microbiome analysis of a Fusarium wilt suppressive soil and a Fusarium wilt conducive soil from the châteaurenard region. Frontiers in Microbiology. 2018; $9,568$.

33. Wei Z, Hu J, Gu YA, Yin SX, Xu YC, Jousset A, Shen QR, Friman VP. Ralstonia solanacearum pathogen disrupts bacterial rhizosphere microbiome during an invasion. Soil Biology and Biochemistry. 2018; 118, 8-17.

34. Zhou D, Jing T, Chen Y, Wang F, Qi D, Feng R, Xie J, Li H. Deciphering microbial diversity associated with Fusarium wilt-diseased and disease-free banana rhizosphere soil. BMC microbiology. 2019; 19(1), 161.

35. Chapelle E, Mendes R, Bakker PA, Raaijmakers JM. Fungal invasion of the rhizosphere microbiome. The ISME journal. 2016; 10(1), 265-268.

36. Bubici G, Kaushal M, Prigigallo Ml, Gómez-Lama Cabanás C, Mercado-Blanco J. Biological control agents against Fusarium wilt of banana. Frontiers in Microbiology. 2019; 10, 616.

37. Xue C, Penton CR, Shen Z, Zhang R, Huang Q, Li R, Shen QR. Manipulating the banana rhizosphere microbiome for biological control of Panama disease. Scientific Reports. 2015; 5, 11124.

38. Wu Q, Sun R, Ni M, Yu J, Li Y, Yu C, Chen J. Identification of a novel fungus, Trichoderma asperellum GDFS1009, and comprehensive evaluation of its biocontrol efficacy. PLoS One. 2017; 12(6), e0179957.

39. Fu L, Ruan YZ, Tao CY, Li R, Shen QR. Continous application of bioorganic fertilizer induced resilient culturable bacteria community associated with banana Fusarium wilt suppression. Scientific Reports. 2016; 6, 27731.

40. Schlatter D, Kinkel L, Thomashow L, Weller D, Paulitz T. Disease suppressive soils. new insights from the soil microbiome. Phytopathology. 2017; 107(11), 1284-1297.

41. Mardones W, Di Genova A, Cortés MP, Travisany D, Maass A, Eyzaguirre J. The genome sequence of the soft-rot fungus Penicillium purpurogenum reveals a high gene dosage for lignocellulolytic enzymes. Mycology. 2018; 9(1), 59-69.

42. Mardones W, Callegari E, Eyzaguirre J. Corncob and sugar beet pulp induce specific sets of lignocellulolytic enzymes in Penicillium purpurogenum. Mycology. 2018; 10(2), 118-125.

43. Varriale S, Houbraken J, Granchi Z, Pepe O, Cerullo G, Ventorino V, Chin-A-Woeng T, Meijer M, Riley R, Grigoriev I V, Henrissat B, de Vries R P, \& Faraco V. Talaromyces borbonicus, sp. nov., a novel fungus from biodegraded Arundo donax with potential abilities in lignocellulose conversion. Mycologia. 2018; 110(2), 316-324.

44. Spink D S, Rowe R C. Evaluation of Talaromyces flavus as a biological control agent against Verticillium dahliae in potato. Plant Disease. 1989; 73(3): 230-236

45. Kim M J, Shim C K, Kim Y K, Hong S J, Park J H, Han E J, Kim S C. Enhancement of seed dehiscence by seed treatment with Talaromyces flavus GG01 and GG04 in ginseng (Panax ginseng). The plant pathology journal. 2017; 33(1), 1-8.

46. Borrero C, Trillas MI, Ordovás José, Tello JC, Avilés Manuel. Predictive factors for the suppression of Fusarium wilt of tomato in plant growth media. Phytopathology. 2004; 94(10), 1094-1101.

47. Gherbawy Y, Elhariry H, Altalhi A, El-Deeb B, Khiralla G. Molecular screening of Streptomyces isolates for antifungal activity and family 19 chitinase enzymes. The Journal of Microbiology. 2012; 50(3), 459-468.

48. Ueki A, Takehara T, Ishioka G, Kaku N, Ueki K. Degradation of the fungal cell wall by clostridial strains isolated from soil subjected to biological soil disinfestation and biocontrol of Fusarium wilt disease of spinach. Applied Microbiology and Biotechnology. 2017; 101(22), 8267-8277.

49. Copete-Pertuz LS, Alandete-Novoa F, Placido J, Correa-Londono GA, Mora-Martinez AL. Enhancement of ligninolytic enzymes production and decolourising activity in Leptosphaerulina sp. by co-cultivation with Trichoderma viride and Aspergillus terreus. Science of the Total Environment. 2019; $646,1536-1545$.

50. Strub C, Dieye CAT, Nguyen PA, Constancias F, Durand N, Guendouz S, Pratlong M, Fontana A, Schorr-Galindo S. Transcriptomes of the interaction between Fusariumverticillioides and a Streptomyces strain reveal the fungal defense strategy under the pressure of a potential biocontrol agent. Fungal Biology. 2019. https://doi: org/10. 1016/j. funbio. 2019. 11. 007.

51. Levin DE. Regulation of cell wall biogenesis in Saccharomyces cerevisiae: the cell wall integrity signaling pathway. Genetics. 2011; 189(4), $1145-1175$.

52. Orlean P. Architecture and biosynthesis of the Saccharomyces cerevisiae cell wall. Genetics, 2012, 192(3), 775-818.

53. López-Fernández L, Ruiz-Roldán C, Pareja-Jaime Y, Prieto A, Khraiwesh H, Roncero MI. The Fusarium oxysporum gnt2, encoding a putative Nacetylglucosaminetransferase, is involved in cell wall architecture and virulence. PLoS One. 2013; 8(12), e84690. 
54. Birkaya B, Maddi A, Joshi J, Free SJ, Cullen PJ. Role of the cell wall integrity and filamentous growth mitogen-activated protein kinase pathways in cell wall remodeling during filamentous growth. Eukaryotic Cell. 2009; 8(8), 1118-1133.

55. Trilla JA, Durán A, Roncero C. Chs7p, a new protein involved in the control of protein export from the endoplasmic reticulum that is specifically engaged in the regulation of chitin synthesis in Saccharomyces cerevisiae. The Journal of Cell Biology. 1999; 145(6), 1153-1163.

56. Lam KK, Davey M, Sun B, Roth AF, Davis NG, Conibear, E. Palmitoylation by the DHHC protein Pfa4 regulates the ER exit of Chs3. The Journal of Cell Biology. 2006; 174(1), 19-25.

57. Roy S, Chiba YM, Jigami Y. Characterization of Yeast Yea4p, a uridinediphosphate-N-acetylglucosamine transporter localized in the endoplasmic reticulum and required for chitin synthesis. Journal of Biological Chemistry. 2000; 275(18), 13580-13587.

58. Leidich SD, Drapp DA, Orlean P. A conditionally lethal yeast mutant blocked at the first step in glycosyl phosphatidylinositol anchor synthesis. Journal of Biological Chemistry. 1994; 269(14), 10193-10196.

59. Pittet M, Conzelmann A. Biosynthesis and function of GPI proteins in the yeast Saccharomyces cerevisiae. BBA - Molecular and Cell Biology of Lipids. 2007; 1771(3), 405-420.

60. Li J, Mouyna I, Henry C, Moyrand F, Malosse C, Chamot-Rooke J, Fontaine T. Glycosylphosphatidylinositolanchors from galactomannan and GPI-anchored protein are synthesized by distinct pathways in Aspergillus fumigatus. Journal of Fungi. 2018; 4(1), 19.

61. Mitsuboshi M, Kioka Y, Noguchi K, Asakawa S. Evaluation of suppressiveness of soils exhibiting soil-borne disease suppression after long-term application of organic amendments by the co-cultivation method of pathogenic Fusarium oxysporum and indigenous soil microorganisms. Microbes and Environments. 2018; 33(1), 58-65.

\section{Figures}

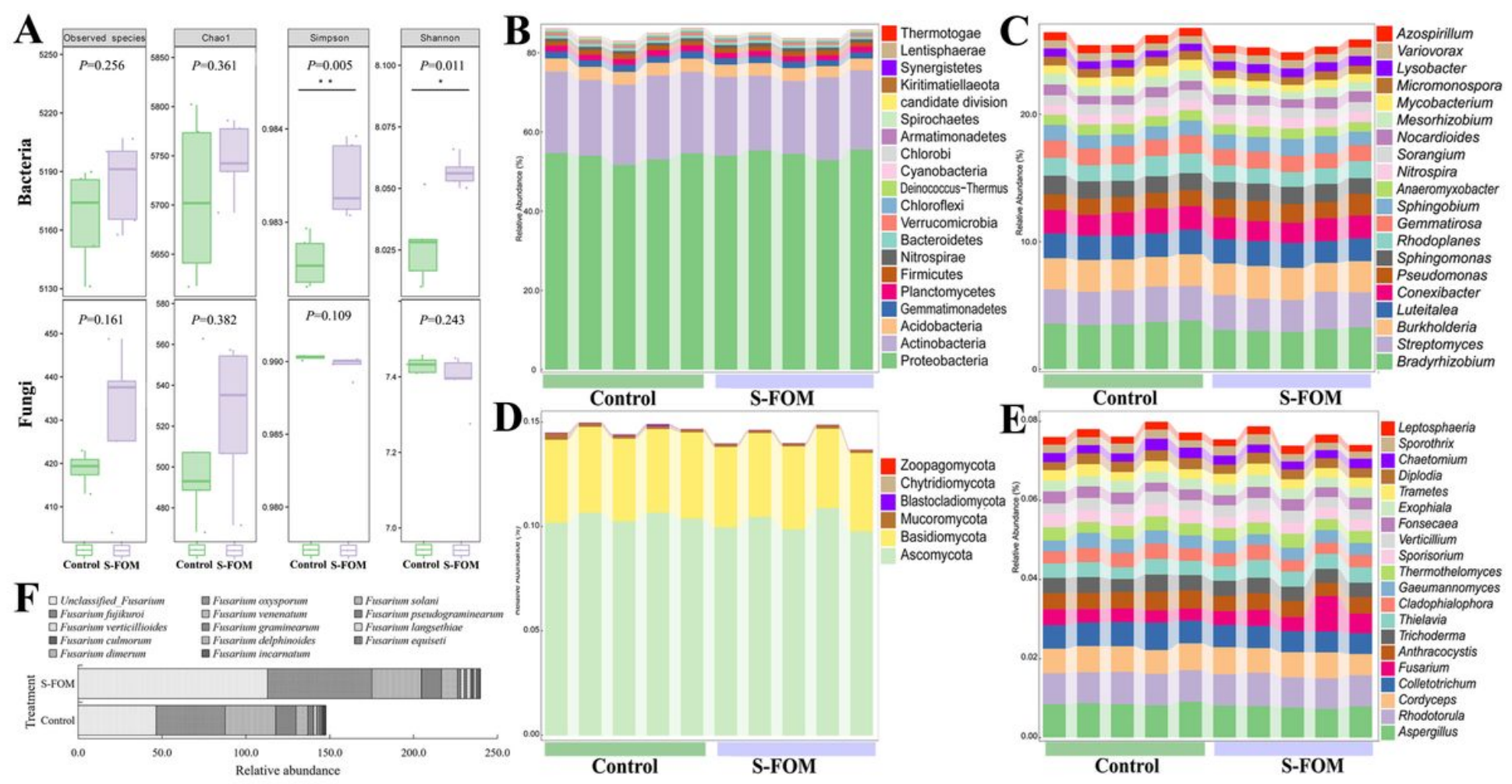

Figure 1

Response of microbial alpha diversity index and community composition to the invasion of F. oxysporum f. sp. momordicae SG-15 based on metagenomics. 


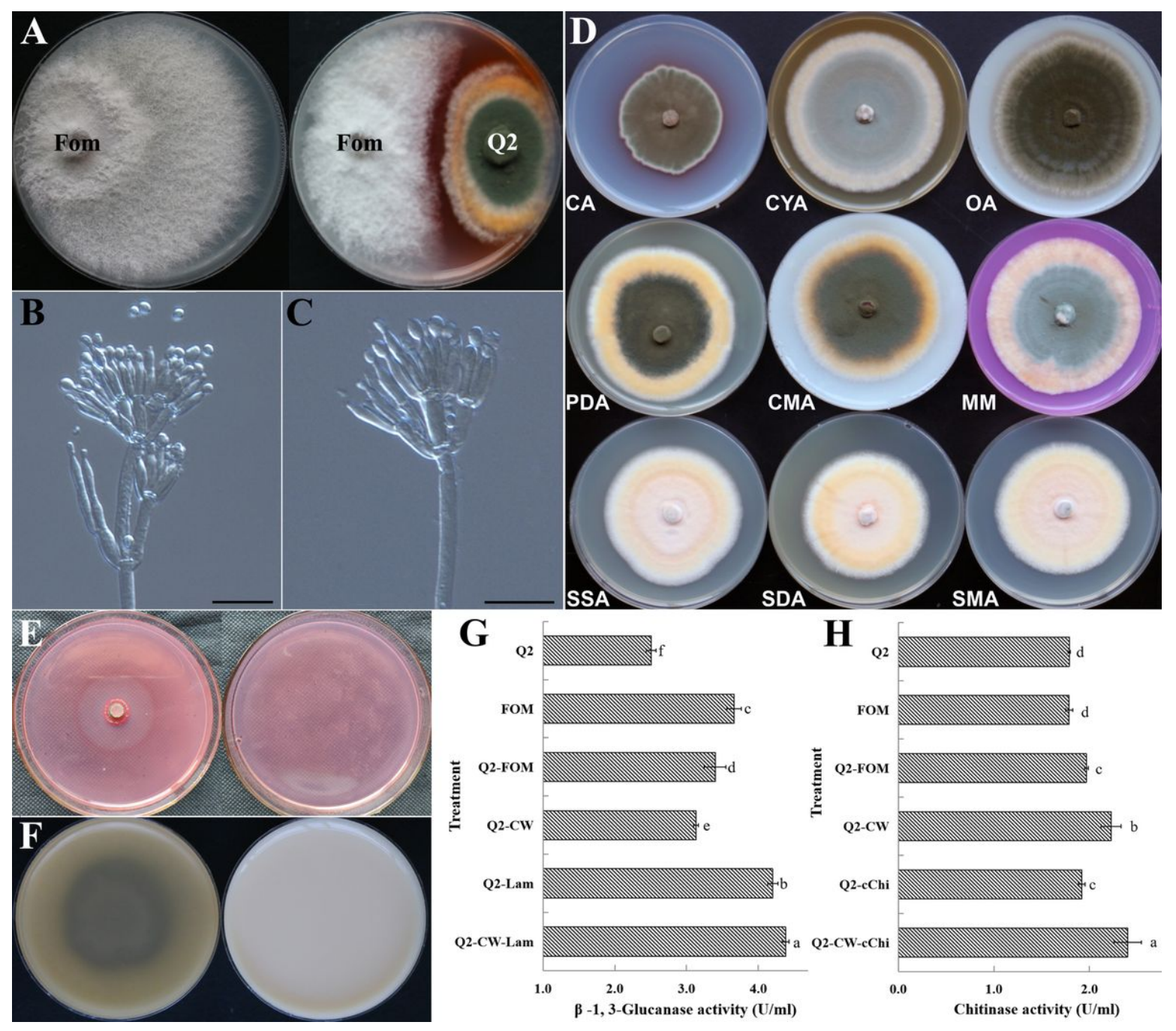

Figure 2

The morphological characteristics and biocontrol function of beneficial microorganisms strain Q2 
A

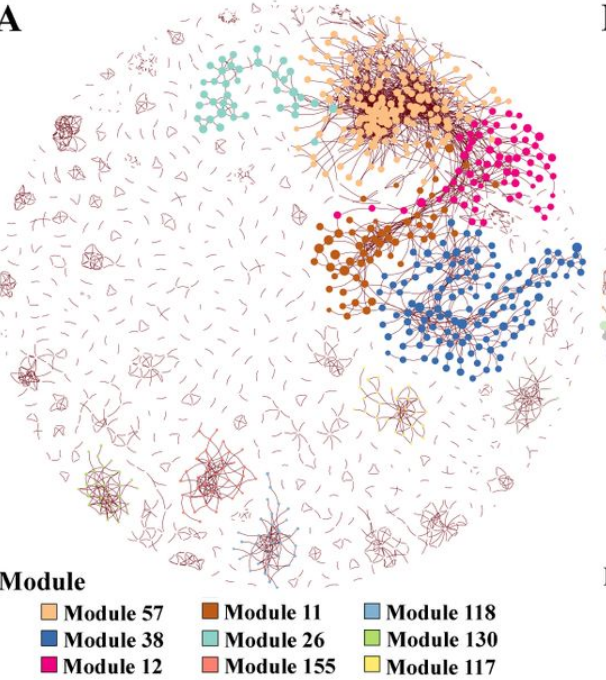

Ciso

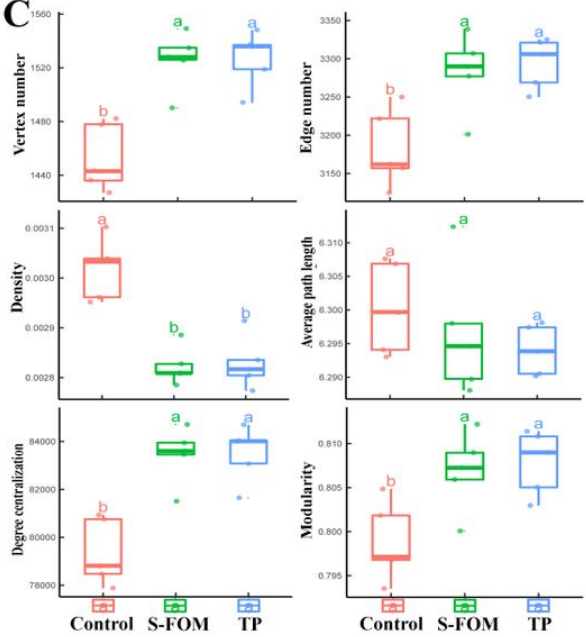

B

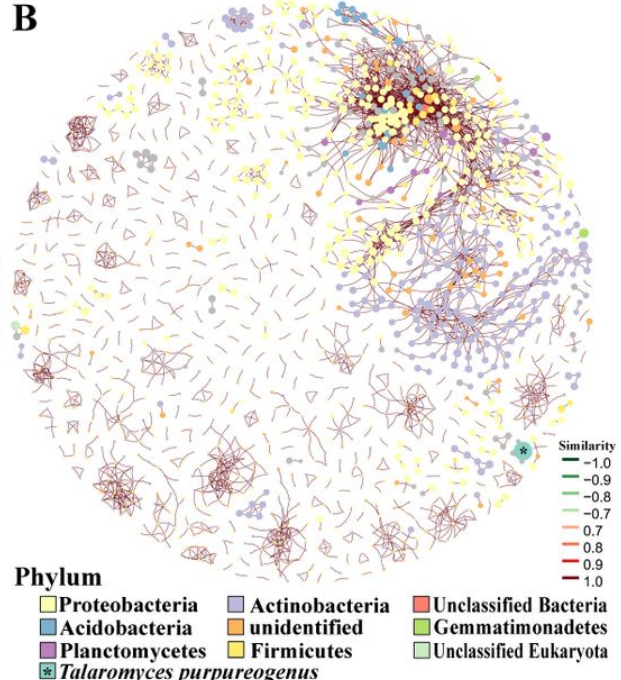

$\mathbf{E}$
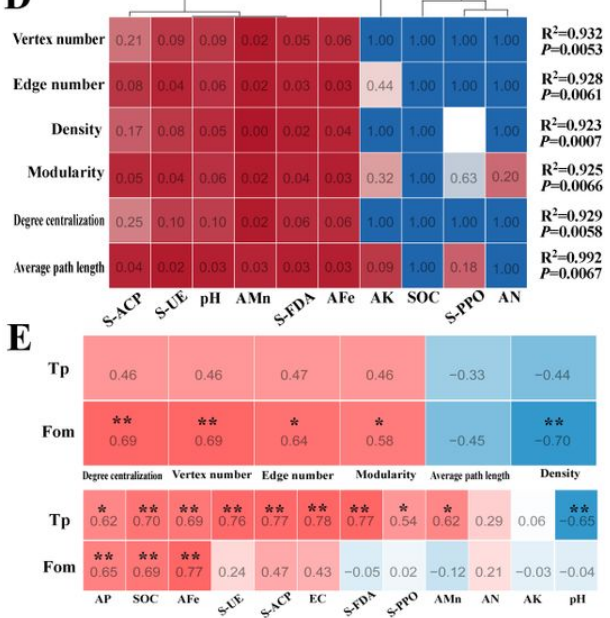

Figure 3

Co-occurrence networks of soil communities constructed from Control, S-FOM and TP based on correlation analysis 


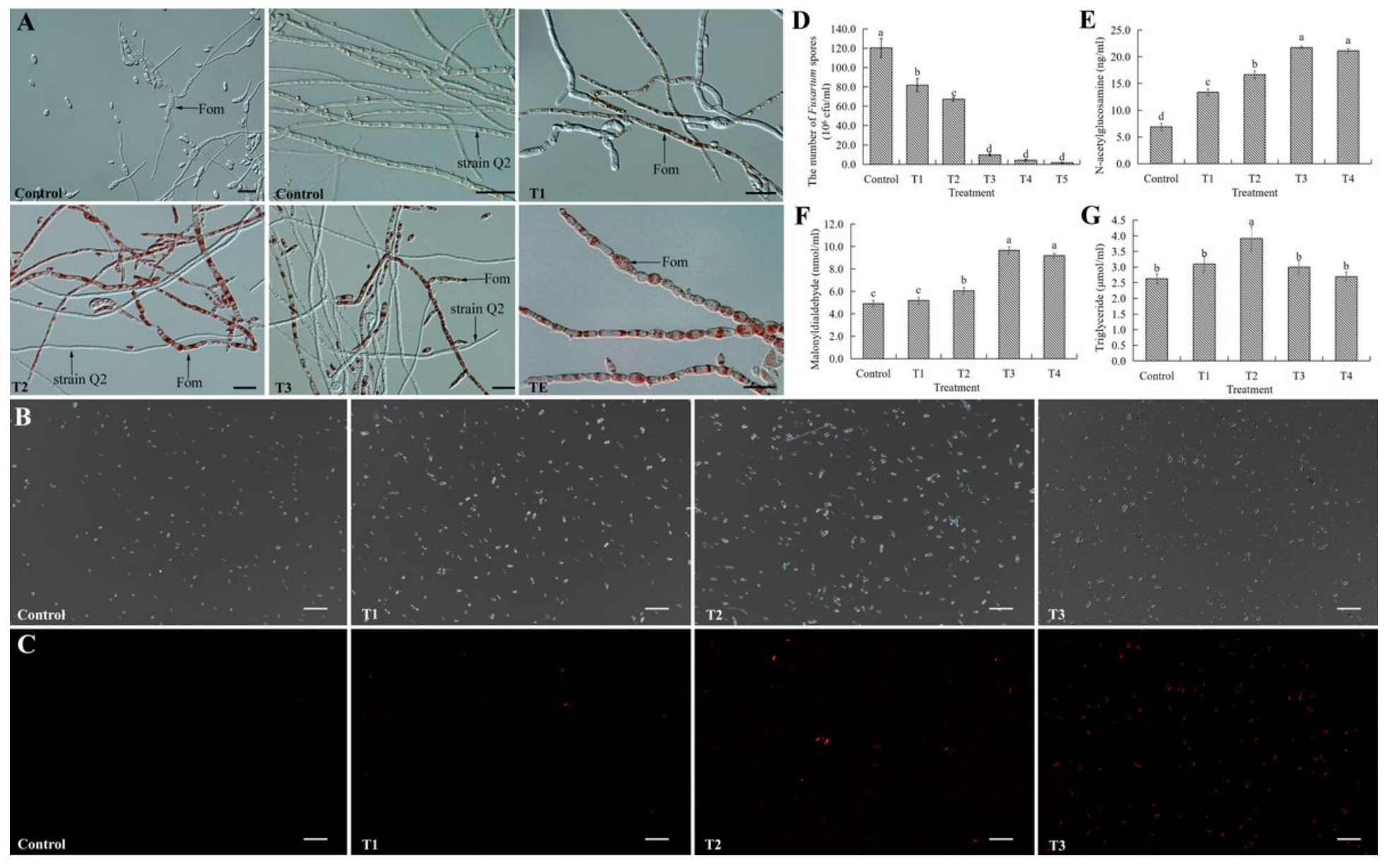

Figure 4

The effects of strain Q2 on the growth and reproduction of Fom during interactions between T. purpureogenus strain Q2 and Fom
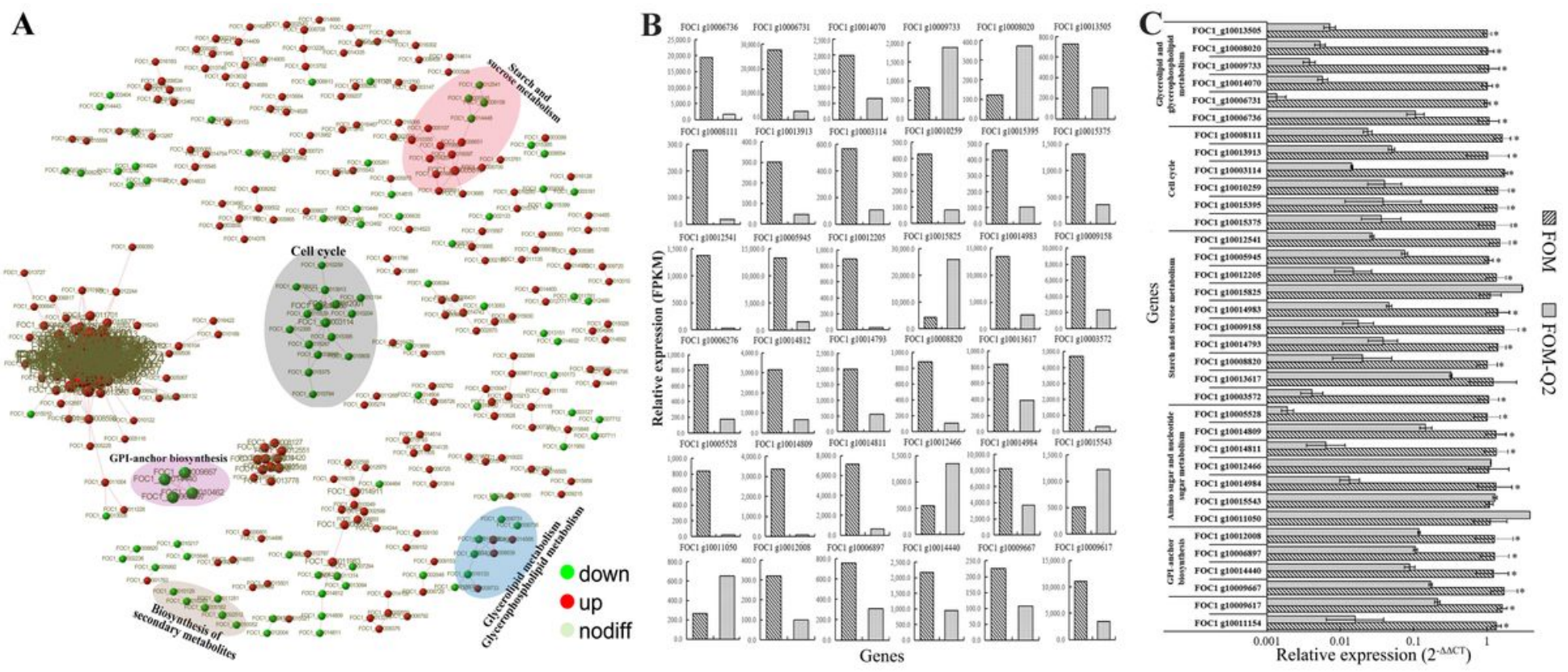

Figure 5

The effects of strain Q2 on the metabolic pathways and related gene expression of Fom during interactions between T. purpureogenus strain Q2 and Fom 

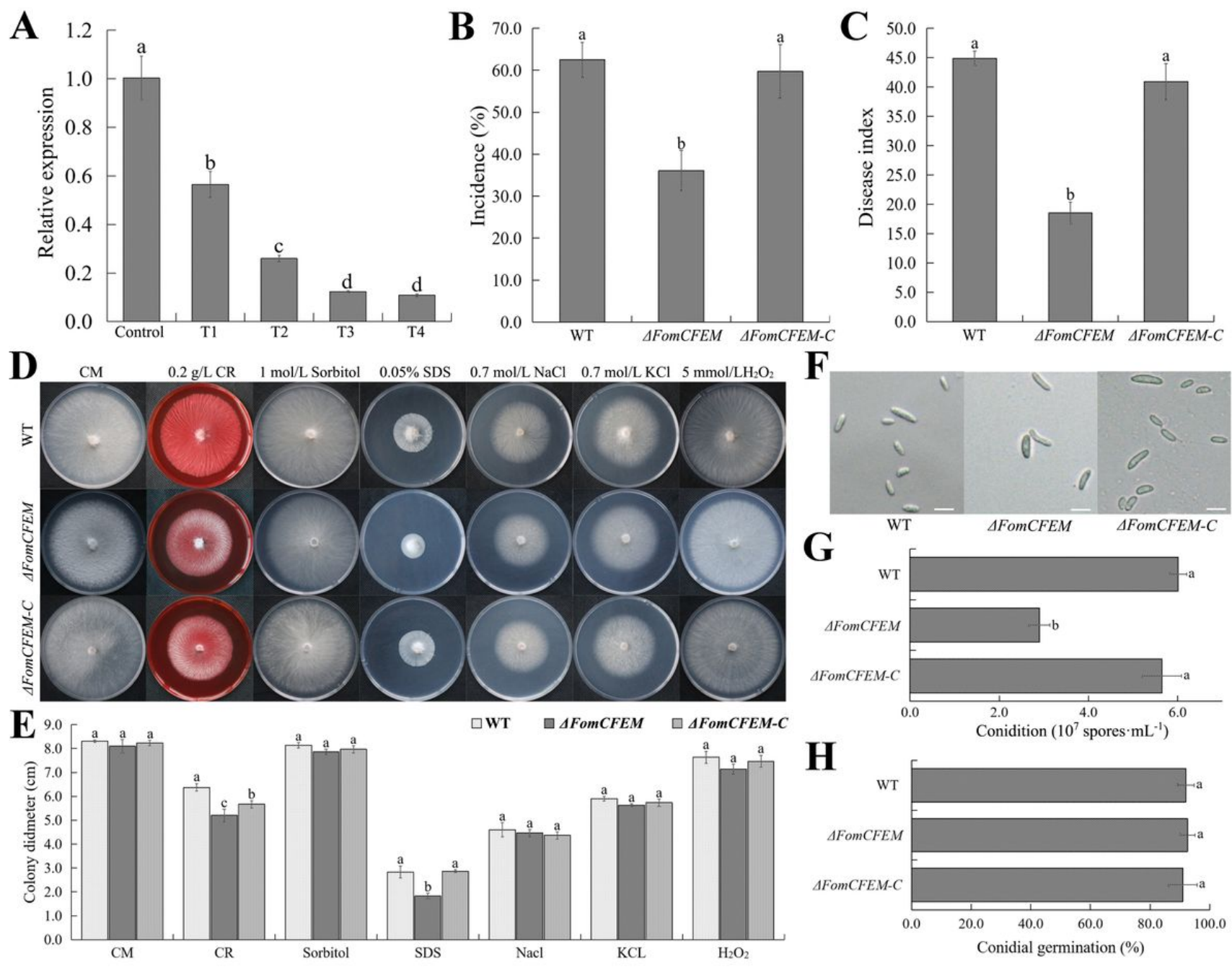

Figure 6

The effects of strain Q2 on the expression of the gene containing a CFEM domain structure (FomCFEM) and the function analysis of FomCFEM

\section{Supplementary Files}

This is a list of supplementary files associated with this preprint. Click to download.

- SupportingInformationFig.S114.docx

- SupportingInformationTableS114.xlsx 